\title{
Scalar oscillatory integrals in smooth spaces of homogeneous type
}

\author{
Philip T. Gressman
}

\begin{abstract}
We consider a generalization of the notion of spaces of homogeneous type, inspired by recent work of Street (2011) on the multi-parameter Carnot-Carathéodory geometry, which endows such spaces with differential structure. The setting allows one to formulate estimates for scalar oscillatory integrals on these spaces which are uniform and respect the underlying geometry of both the space and the phase function. As a corollary we obtain a generalization of a theorem of Bruna, Nagel, and Wainger (1988) on the asymptotic behavior of scalar oscillatory integrals with smooth, convex phase of finite type.
\end{abstract}

\section{Introduction}

Given a manifold $\Omega$ and a measure of smooth density $\mu$, a common problem in analysis is to establish an estimate for scalar oscillatory integrals of the form

$$
\int_{\Omega} e^{i f} \psi d \mu
$$

where the phase $f$ is real-valued and the amplitude $\psi$ is supported on a set of finite measure. We would like the estimates to be uniform in $f$ and $\psi$ and to effectively reduce the problem of estimating this integral to a sublevel set problem for the gradient of $f$ (since the method of stationary phase dictates that there will be substantial cancellation away from the critical points of $f$ ). Ideally, this objective should be accomplished in a geometrically invariant way, if at all possible, although to date this has proven to be a difficult task to accomplish, especially in dimension greater than one. We would also like to assume as little as possible about $\psi$, as it is generally regarded to be of secondary importance when contrasted with the phase.

A tremendous amount of work has already been devoted to understanding integrals of the form (1.1) and related objects in higher dimensions. If one is willing

Mathematics Subject Classification (2010): Primary 41A60; Secondary 28C99, 51H25.

Keywords: Oscillatory integrals, spaces of homogeneous type, stationary phase. 
to compromise somewhat on uniformity requirements (and, for example, restrict attention to phases $f$ which are scalar multiples of a single fixed phase function $\Phi$ or simple perturbations of such phases), methods based on resolution of singularities provide extremely powerful tools for understanding (1.1). The seminal result in this direction is due to Varčenko [25]. The history of this field is lengthy and we will not try to summarize it here, but we will note that the emphasis of some of the most recent work, due to Collins, Greenleaf, and Pramanik [6], as well as Greenblatt [8], has been to produce resolution of singularities algorithms which are much more concrete (and more easily applied by nonspecialists) than were those previously available; these new algorithms are also able to handle phase functions which fall outside the scope of earlier work for technical reasons. Despite the power of these methods, answering questions of uniformity by means of resolution of singularities is still generally a difficult task. It is also worth noting that, somewhat paradoxically, resolution of singularities methods tend to encounter added difficulties when the decay rate of scalar oscillatory integrals is relatively high. Work in the complementary direction, emphasizing uniformity of some form or another (and sacrificing sharpness of the estimates when necessary) also abounds: see, for example, Carbery, Christ, and Wright [3]; Carbery and Wright [4]; Greenblatt [9]; Iosevich [13]; Ikromov, Kempe, and Müller [12]; Karpushkin [14]; Phong and Stein [16]; Phong, Stein, and Sturm [17], [18]; Phong and Sturm [19]; Pramanik and Yang [20]; Rogers [21]; Seeger [22]; and the author's [10], [11]. The goals, ideas, and methods to be found in these results are numerous and diverse.

If we further narrow attention to uniform estimates of (1.1) which display some degree of geometric invariance, one is quickly left with a rather short list of known results. Of these we highlight the work of Bruna, Nagel, and Wainger [1], in which they succeed in estimating the decay of the Fourier transform of a smooth, convex surface of finite type in terms of the volume growth rates of surface caps. In a similar spirit, the goal of the present paper is to establish a uniform estimate for scalar oscillatory integrals in terms of some geometrically defined, nonoscillatory quantity. In contrast to Bruna, Nagel, and Wainger, the nonoscillatory quantity we identify is not subject to convexity or strict finite type assumptions.

When the phase is not convex, surface caps are not necessarily meaningful, since sublevel sets of the phase can be much too large to be a useful substitute for a sharp, uniform estimate (one can immediately check that the estimating the oscillatory integral with phase $x y$ on $[0,1]^{2}$ in terms of sublevel sets of $x y$ gives a superfluous factor of $\log \frac{1}{|\lambda|}$ compared to the usual stationary phase argument). Thus it is natural to look for some sharper substitute for the sublevel set which reduces to the previous case when the phase is known to be convex. This paper establishes such an estimate by generalizing the notion of spaces of homogeneous type and estimating oscillatory integrals on such spaces in terms of nonoscillatory integrals which play the role of the surface caps. The resulting estimate (1.9) can be thought of as a self-contained, geometric reformulation of oscillatory integrals into nonoscillatory integrals. Corollary 1.2 shows how the estimate (1.9) can function as a black-box replacement for the delicate integration-by-parts arguments and estimates (e.g., stationary phase) of Bruna, Nagel, and Wainger. The broader 
hope of developing (1.9) is that it may serve the same sort of black-box role in future estimates of more complicated objects as well, for example, oscillatory integral operators in higher dimensions generalizing the work of Phong, Stein, and Sturm [18].

The generalization of spaces of homogeneous type, a concept originally due to Coifman and Weiss [5], introduced here endows them with a compatible differential structure. That such a generalization is possible was already observed in some sense by Bruna, Nagel, and Wainger; however, the important point here is that the construction can be completely divorced from a fine-structure analysis of the phase and does not, for example, require convexity. Using this new structure, we establish a natural estimate of (1.1) which is both uniform and firmly tied to the underlying geometry of the manifold and the phase. The new smoothness hypotheses added to spaces of homogeneous type are intuitive and, for the most part, have already been shown to hold in many of the familiar cases. In particular, the smoothness hypotheses are satisfied in Carnot-Carathéodory geometries, as is shown in the series of papers beginning with Nagel, Stein, and Wainger [15]; including Tao and Wright [24]; and culminating with Street [23].

We begin by assuming only that $\Omega$ is a topological space and $\mu$ is a Borel measure on $\Omega$. The familiar axioms associated with spaces of homogeneous type begin with a family of balls $B_{j}(x) \subset \Omega$. One's intuition should be that the balls are geometrically nice sets containing $x$, but note that we will explicitly avoid the assumption they are open. Here $x$ may be any point in $\Omega$, and in this paper the index $j$ will be contained in $\mathbb{Z}^{d}$; this should be thought of as corresponding to Street's "multiparameter" setting for Carnot-Carathéodory geometry (although the main theorems of this paper have interesting new consequences even in the case $d=1$ corresponding to single parameter geometry). For technical reasons, we will allow the possibility that $B_{j}(x)=\emptyset$ for certain values of the parameter $j$; we will say that such balls do not exist or are not defined. In all other cases, (i.e., when $\left.B_{j}(x) \neq \emptyset\right)$, it will be required that $x \in B_{j}(x)$. Regarding the index $j \in \mathbb{Z}^{d}$ (referred to as the scale of the ball), we will use the standard notation that $j^{\prime} \leq j$ when the corresponding inequality holds for each coordinate of $j^{\prime}$ and $j$, and we define $\left|j-j^{\prime}\right|$ to be the $\ell^{\infty}$-norm of $j-j^{\prime}$ on $\mathbb{Z}^{d}$. We will also identify the integers $\mathbb{Z}$ with the diagonal subset of $\mathbb{Z}^{d}$, i.e., $n=(n, \ldots, n)$.

The (mostly) familiar assumptions regarding the geometry of these balls are recorded here. We suppose that for some open $\Omega_{0} \subset \Omega$, we have the following:

(i) (Compatibility) If $B_{j^{\prime}}\left(x^{\prime}\right) \cap B_{j}(x) \neq \emptyset$ for some $x, x^{\prime} \in \Omega_{0}$ and $j, j^{\prime} \in \mathbb{Z}^{d}$, then $B_{j-1}\left(x^{\prime}\right)$ exists.

(ii) (Engulfing) Suppose $B_{j}(x)$ is defined for some $x \in \Omega_{0}$ and $j \in \mathbb{Z}^{d}$. If there exist $x^{\prime} \in \Omega_{0}$ and $j^{\prime} \leq j-1$ such that $B_{j-1}(x) \cap B_{j^{\prime}}\left(x^{\prime}\right) \neq \emptyset$, then $B_{j^{\prime}}\left(x^{\prime}\right) \subset$ $B_{j}(x)$.

(iii) (Weak doubling) There is a finite constant $C$ such that any distinct points $x_{1}, \ldots, x_{N}$ of $\Omega_{0}$ and any index $j \in \mathbb{Z}^{d}$ with $B_{j}\left(x_{k}\right) \cap B_{j}\left(x_{l}\right)=\emptyset$ for all $k \neq l$ have the property that at most $C$ of these points satisfy $B_{j+1}(x) \cap B_{j+1}\left(x_{k}\right) \neq \emptyset$ for any fixed $x \in \Omega_{0}$. 
In particular, note that whenever a ball $B_{j}(x)$ is defined, one automatically knows that $B_{j-1}(x)$ exists and is smaller than $B_{j}(x)$. Next we impose additional smoothness hypotheses. For each nonempty ball $B_{j}(x)$, we assume that there is a homeomorphism $\Phi_{j, x}: \mathbb{B}^{d_{x}} \rightarrow B_{j}(x)$ which maps 0 to $x$, where $\mathbb{B}^{d_{x}}$ is the open Euclidean unit ball in dimension $d_{x}$. The dimension $d_{x}$ may depend on $x$, but we assume that any two balls which intersect have the same dimension. We will also assume that the supremum over $x$ of $d_{x}$ is finite; it will be referred to as the dimension when no confusion will arise. We will abuse notation and use $\mathbb{B}$ to refer to the unit ball in Euclidean space of the appropriate dimension (depending on context). In a nutshell, we will assume that these homeomorphisms are smooth with respect to each other when compared on two comparable balls. Specifically we assume:

(iv) (Smooth nesting) For some universal $c<1$,

$$
\sup _{t \in \mathbb{B}}\left|\Phi_{j, x}^{-1} \circ \Phi_{j-1, x}(t)\right| \leq c .
$$

(v) (Smooth engulfing) If $B_{j}(x) \cap B_{j^{\prime}}\left(x^{\prime}\right) \neq \emptyset$, then there is a constant $C_{\left|j-j^{\prime}\right|}$ depending only on $\left|j-j^{\prime}\right|$, the dimension, and $m$, such that

$$
\left|\partial_{t}^{\alpha}\left[\Phi_{j, x}^{-1} \circ \Phi_{j^{\prime}, x^{\prime}}(t)\right]\right| \leq C_{\left|j-j^{\prime}\right|}
$$

uniformly as $t$ ranges over $\Phi_{j^{\prime}, x^{\prime}}^{-1}\left(B_{j}(x) \cap B_{j^{\prime}}\left(x^{\prime}\right)\right)$ and $\alpha$ ranges over all multiindices of order at most $m$.

Under these assumptions, it is possible to quantify the smoothness of a function $f$ at any particular point $x$ and any given scale $j$. We define

$$
\left|d_{x}^{k} f\right|_{j}:=\sup _{1 \leq|\alpha| \leq k}\left|\partial_{t}^{\alpha}\left[f \circ \Phi_{j, x}(t)\right]\right|_{t=0} \mid
$$

for any $k=1, \ldots, m$. We also denote $\left|d_{x}^{1} f\right|_{j}$ by $\left|d_{x} f\right|_{j}$ when no confusion will arise. Under the assumptions above, the quantity $\left|d_{x} f\right|_{j}$ satisfies a sort of weak differential invariance property: namely, that compositions of $f$ with quantitatively "nice" diffeomorphisms (meaning, for example, that compositions $\Phi_{j, x}$ for each $j$ and $x$ have uniformly bounded derivatives through some high order) will preserve the magnitude of $d_{x} f$ at scale $j$ up to a bounded factor. That this sort of weak invariance is the best that can be hoped for can be seen by considering the effect of a rough (in this case, $C^{1}$ ) change of variables in the integral (1.1). If such a rough transformation is made, the value of the integral will remain constant, but the method of stationary phase will fail for technical reasons (because the integration by parts argument in stationary phase cannot be justified unless the derivatives of the phase are of bounded variation). Thus the only way to deduce decay for such an integral would to understand the very precise coincidence of the irregularity of both $f$ and $\psi$. Typically for applications one would like to assume as little as possible about the function $\psi$. Thus we are necessarily constrained to consider only the effect of nice diffeomorphisms.

The final set of assumptions we make concern the (Borel) measure $\mu$ and its regularity with respect to the balls $B_{j}(x)$. To this end, a set $L \subset \Omega$ is called a leaf when it has the following properties: 
1. Every ball $B_{j}(x)$ with $x \in \Omega_{0}$ which intersects $L$ is contained in $L$.

2. Every ball contained in $L$ is relatively open in $L$.

3. $L$ has a countable dense subset.

We will assume that $\Omega$ is equipped with a measure $\mu$ which may be "factored" onto the leaves in the following sense:

(vi) (Regularity of measure) There is a collection of leaves $\mathcal{F}$ (i.e., a foliation) with measure $\mu_{\mathcal{F}}$ and Borel measures $\mu_{L}$ on each leaf $L \in \mathcal{F}$ such that

$$
\int_{\Omega} f d \mu=\int_{\mathcal{F}}\left[\int_{L} f d \mu_{L}\right] d \mu_{\mathcal{F}}(L)
$$

for all Borel measurable functions $f$ on $\Omega$. Each measure $\mu_{L}$ should have smooth density, meaning that for any leaf $L$ and any ball $B_{j}(x) \subset L$ with $x \in \Omega_{0}$ and $\mu_{L}\left(B_{j}(x)\right) \neq 0$, there is a nonvanishing function $J_{j, x}$ such that

$$
\frac{1}{\mu_{L}\left(B_{j}(x)\right)} \int_{B_{j}(x)} f d \mu_{L}=\int_{\mathbb{B}} f \circ \Phi_{j, x}(t) J_{j, x}(t) d t
$$

with $\left\|J_{j, x}\right\|_{C^{m}(\mathbb{B})} \leq C$ and $\inf _{t \in \mathbb{B}} J_{j, x}(t) \geq c$ uniformly in $j, x$, and $L$.

We must make the technical assumption that the balls $B_{j}(x)$ are Borel measurable and the maps

$$
x \mapsto \Phi_{j, x}(u)
$$

for fixed $j$ and $u$ are defined on a Borel measurable set and are Borel measurable functions there. It is perhaps worth emphasizing once again that Street has verified these axioms already in the Carnot-Carathéodory geometry (this will be discussed in detail in the final section). The main theorem may can be stated as follows:

Theorem 1.1. Assume that (i) through (vi) hold. Fix any $\epsilon \in(0,1)$, and suppose $m \geq 2$. Let $E \subset \Omega_{0}$ consist of those points where $d_{x} f \neq 0$, and suppose $R: E \rightarrow \mathbb{Z}^{d}$ is some Borel measurable function such that $B_{R(x)+1}(x)$ is well defined for each $x \in E$ and the conditions

$$
\begin{aligned}
B_{R(x)}(x) \cap B_{R(y)}(y) & \neq \emptyset \Rightarrow|R(x)-R(y)| \lesssim 1, \\
\left|d_{x}^{m} f\right|_{R(x)} & \lesssim \sum_{k=1}^{m-1} \epsilon^{k-m}\left|d_{x}^{k} f\right|_{R(x)}, \\
\sup _{y \in B_{R(x)}(x)} \epsilon\left|d_{y} f\right|_{R(y)} & \lesssim 1+\inf _{y \in B_{R(x)}(x)} \epsilon\left|d_{y} f\right|_{R(y)},
\end{aligned}
$$

hold with implicit constants uniform with respect to $x, y \in E$ and $\epsilon$. Then for any smooth, bounded $\psi$ whose support has finite measure in $\Omega_{0}$, there is another function $\psi_{m}$ such that

$$
\int_{\Omega_{0}} e^{i f} \psi d \mu=\int_{\Omega_{0}} e^{i f} \psi_{m} d \mu
$$


and

$$
\left|\psi_{m}(x)\right| \lesssim \frac{\sum_{k=0}^{m-1} \epsilon^{k}\left|d_{x}^{k} \psi(x)\right|_{R(x)}}{\left(1+\epsilon\left|d_{x} f(x)\right|_{R(x)}\right)^{m-1}}
$$

In particular, one has the inequality

$$
\left|\int_{\Omega_{0}} e^{i f} \psi d \mu\right| \lesssim \int_{\Omega_{0}} \frac{\sum_{k=0}^{m-1} \epsilon^{k}\left|d_{x}^{k} \psi(x)\right|_{R(x)}}{\left(1+\epsilon\left|d_{x} f(x)\right|_{R(x)}\right)^{m-1}} d \mu(x) .
$$

Informally, the theorem establishes a more geometric version of the method of stationary phase, meaning that the usual gradient factor appears in the denominator, but only when measured at the correct scale at each point. Specifically, the reader should think of the right-hand side of (1.9) as measuring the volumes of sublevel sets of $\left|d_{x} f(x)\right|_{R(x)}$, i.e., the magnitude of the gradient when measured at some appropriate scale at each point. The condition (1.5) can be thought of as a sort of Lipschitz condition for the scales (meaning that if one measures on a very fine scale at $x$, one must also measure at fine scales when relatively near $x$ ). The inequality (1.6) plays the role of a finite-type condition, but it should not be understood literally as such, since it only holds on balls away from the set where $d_{x} f=0$. In particular, in most applications an $\epsilon$ is guaranteed to exist satisfying (1.6) uniformly provided only that the ball $B_{R(x)}(x)$ is sufficiently small at each point. (Note that, when the derivatives of $\psi$ satisfy some strong a priori bound, one benefits the most in (1.8) by taking $\epsilon$ as large as possible, namely, comparable to 1.) Elementary examples show that this can, in fact, be achieved in many cases when $f$ is not of finite type.

Although Theorem 1.1 may seem far-removed from the surface cap example for convex phases, it will be shown that the original inequality of Bruna, Nagel, and Wainger is, in fact, a rather straightforward corollary of (1.9). (When combined with a uniform estimate of Carbery [2], Theorem 1.1 also generalizes earlier work of Cowling and Mauceri [7] on weighted estimates, but the details of this argument are left to the reader.) Moreover, Theorem 1.1 provides two key improvements of this earlier work. The first is that we will have a slightly stronger sort of uniformity than was originally available; we will explicitly identify quantities that determine the values of the implicit constants and we will not, for example, assume that the domain of integration is compact. We will also explicitly identify the number of derivatives necessary for estimates to hold (so one need not actually assume that the convex phase is $C^{\infty}$ ). The second (and perhaps more interesting) extension is that the finite-type assumption will be replaced by a strictly weaker one which in some cases includes convex functions which are not finite type (and even phases not strictly convex). In particular, the relaxation of these two conditions includes new phases (corresponding, for example, to new Fourier decay estimates for new surfaces) which have not previously been treated elsewhere.

We begin by identifying the substitute notion of regularity we will employ. Let $f \in C^{m}([0, T])$ for some $m \geq 2$ be a nonnegative convex function with $f(0)=0$ and $f^{\prime}(0) \geq 0$. We say that $f$ is tame on $[0, T]$ to order $m$ when there is a 
constant $C<\infty$ such that for each $k=2, \ldots, m$ and each $t \in[0, T]$ we have

$$
|f(t)|^{k-1}\left|f^{(k)}(t)\right| \leq C\left|f^{\prime}(t)\right|^{k}
$$

Although the tameness inequality may at first seem unusual, it is a natural way to measure regularity of $f$ in a way that is invariant under rescaling the magnitude of $f$ and rescaling the time parameter $t$; for any positive $\alpha$ and $\beta$, the same constant $C$ will be possible for the corresponding inequalities applied to the function $g(t):=$ $\alpha f\left(\beta^{-1} t\right)$ on the interval $[0, \beta T]$. In fact, we may reinterpret (1.10) as follows: if the horizontal and vertical axes of the graph of $f$ are rescaled in such a way that the rescaled function $g$ satisfies $g\left(t_{0}\right)=g^{\prime}\left(t_{0}\right)=1$ at some point $t_{0}$, then the higher derivatives of $g$ at $t_{0}$ (and, hence, the coefficients of the Taylor polynomial at $t_{0}$ ) will be controlled by the constant $C$.

Two important features of (1.10) should be highlighted at this point. The first is that for a polynomial $f$, the constant $C$ appearing in (1.10) can be taken to depend only on the degree of $f$ (a fact which shall be addressed explicitly in section 4.2). The second is that (1.10) does not imply that $f$ is finite type (or even strictly convex). For example, a trivial induction argument establishes that $f(t):=e^{-\left(1+\alpha^{-1}\right) t^{-\alpha}}$ is tame to any finite order on $[0,1]$, for any positive $\alpha$.

Definitions now established, we have the following corollary of Theorem 1.1:

Corollary 1.2. Suppose $f$ is a convex function on an open convex set $\Omega \subset \mathbb{R}^{d}$ containing the origin. Assume that $f$ and its gradient vanish at the origin. If $f$ is uniformly radially tame to order $m$ on $\Omega$ (meaning that $f$ is tame with the same constant on all rays beginning at the origin), then for any compactly supported $\psi \in C^{m}(\Omega)$ and any real $\lambda$,

$$
\left|\int_{\Omega} e^{i \lambda f(x)}(f(x))^{\ell} \psi(x) d x\right| \lesssim \int_{\Omega} \frac{|f(x)|^{\ell} \sum_{k=0}^{m-1}\left|(x \cdot \nabla)^{k} \psi(x)\right|}{(1+|\lambda f(x)|)^{m-1}} d x,
$$

with implicit constant depending only on $m, d$, the nonnegative integer $\ell$, and the constant of uniform radial tameness.

The proof of the corollary is contained in Section 4.2 along with two different propositions which establish stable estimates for the constant of uniform radial tameness (the first proposition dealing with polynomials as promised, and the second establishing finiteness of the constant for convex functions of finite type in the sense of [1]). The final result in Section 4.2 demonstrates how estimates of the sort established by Bruna, Nagel, and Wainger in terms of volumes of caps can be deduced in the standard way from (1.11).

The structure of the rest of this paper is as follows. Section 2 establishes a few immediate observations and is then devoted to a brief study of functions of finite type on Euclidean balls. Though relatively elementary, this section includes a concrete construction on the Euclidean ball of analogues of the classical Littlewood-Paley projections (which eliminates problems near the boundary that occur in more straightforward approaches) which may be of independent interest. 
Theorem 2.1 in this section can be thought of as a sharp characterization of functions of finite type on the ball. By sharp we mean that its main hypothesis (2.4) is implied (with a slightly worse constant) by and substantially weaker than the main conclusion (2.5). Section 3 contains the main covering lemma and the proof of Theorem 1.1. Although the proof of Theorem 1.1 is admittedly technical, the hope is that interested readers will be inclined to avoid such technicalities in the future by appealing to the theorem itself rather than its proof and thereby have the flexibility to think about oscillatory integrals in purely geometric terms rather than thinking of these objects at the usual level of integration by parts arguments. Finally, Section 4 is devoted to three topics. The first regards the choice of scale function $R$ in theorem 1.1. We show that, in many contexts, there is a natural choice for $R$ and that, roughly speaking, it can be thought of as simply the largest scale on which the magnitude of the gradient looks roughly constant. The second topic in Section 4 is the proof of Corollary 1.2 and an analysis of uniform radial tameness. Finally we remind the readers of the relevant definitions, theorems, and inequalities from Street [23] and present the final theorem, Theorem 4.3, which illustrates how the structures behind Theorem 1.1 arise naturally in the context of Carnot-Carathéodory geometry.

\section{Initial steps}

\subsection{Basic observations}

When $B_{j^{\prime}}\left(x^{\prime}\right) \subset B_{j}(x)$, the mapping $\Phi_{j, x}^{-1} \circ \Phi_{j^{\prime}, x^{\prime}}$ is a smooth map from the ball $\mathbb{B}$ into itself. The smooth engulfing property implies (via the Leibniz and chain rules) that

$$
\left\|f \circ \Phi_{j, x}^{-1} \circ \Phi_{j^{\prime}, x^{\prime}}\right\|_{C^{m}(\mathbb{B})} \lesssim\left(1+C_{\left|j-j^{\prime}\right|}\right)^{m}\|f\|_{C^{m}(\mathbb{B})},
$$

with an implied constant depending only on the dimension and $m$ (where $C_{\left|j-j^{\prime}\right|}$ is the same constant appearing in (1.2)). More generally, suppose $f$ is a smooth function on $\Omega$. Fix a point $x_{0} \in \Omega_{0}$. For any ball $B_{j}(x)$ containing $x_{0}$ with $x$ also in $\Omega_{0}$, we may measure the smoothness of $f$ at $x_{0}$ by means of the formula

$$
\left|d_{x_{0}}^{k} f\right|_{B_{j}(x)}:=\sup _{1 \leq|\alpha| \leq k}\left|\partial_{t}^{\alpha}\left[f \circ \Phi_{j, x}(t)\right]\right|_{t=\Phi_{j, x}^{-1}\left(x_{0}\right)} \mid,
$$

that is, by taking the usual mixed partials on the Euclidean ball $\mathbb{B}$ and evaluating at the appropriate point (note that the definition (1.3) corresponds to the case $x=x_{0}$, meaning $\left.\left|d_{x}^{k} f\right|_{j}=\left|d_{x}^{k} f\right|_{B_{j}(x)}\right)$. Although the magnitude of $d f_{x_{0}}$ depends on the choice of the ball $B_{j}(x)$, smooth engulfing will dictate the comparability condition

$$
\left(1+C_{\left|j-j^{\prime}\right|}\right)^{-m}\left|d_{x_{0}}^{k} f\right|_{B_{j^{\prime}}\left(x^{\prime}\right)} \lesssim\left|d_{x_{0}}^{k} f\right|_{B_{j}(x)} \lesssim\left(1+C_{\left|j-j^{\prime}\right|}\right)^{m}\left|d_{x_{0}}^{k} f\right|_{B_{j^{\prime}}\left(x^{\prime}\right)}
$$

whenever $\left|d_{x_{0}}^{k} f\right|_{B_{j}(x)}$ and $\left|d_{x_{0}}^{k} f\right|_{B_{j^{\prime}}\left(x^{\prime}\right)}$ are well defined (with an implicit constant depending only on the usual suspects of dimension and $m$ ). 
One final elementary observation is that when $x \in \Omega_{0}$ and $B_{j}(x) \neq \emptyset$, there is a smooth, nonnegative function $\eta_{j, x}$ supported on $B_{j}(x)$ (meaning it is identically zero outside this ball) and bounded above by one, that is identically one on $B_{j-1}(x)$ and satisfies

$$
\left\|\eta_{j, x} \circ \Phi_{j^{\prime}, x^{\prime}}\right\|_{C^{m}(\mathbb{B})} \lesssim\left(1+C_{\left|j-j^{\prime}\right|}\right)^{m}
$$

for any ball $B_{j^{\prime}}\left(x^{\prime}\right)$. The function $\eta_{j, x}$ is not necessarily smooth on $\Omega$, but is nevertheless Borel measurable. These assertions follow immediately from smooth doubling: choose any $C^{\infty}$ function $\eta$ on the ball $\mathbb{B}$ which is identically zero outside the ball of radius $(1+c) / 2$ centered at the origin, identically one on the ball of radius $c$ centered at the origin, and maps into $[0,1]$. We define $\eta_{j, x}\left(x_{0}\right):=\eta \circ \Phi_{j, x}^{-1}\left(x_{0}\right)$ when $x_{0} \in B_{j}(x)$ and $\eta_{j, x}\left(x_{0}\right)=0$ when $x^{\prime} \notin B_{j}(x)$. By construction we have uniform control on $\left|d_{x_{0}}^{k} \eta_{j, x}\right|_{B_{j^{\prime}}\left(x^{\prime}\right)}$ (as it depends only on $\eta$ ) when $x_{0} \in B_{j}(x)$. In all other cases, $\eta_{j, x}\left(x_{0}\right)$ and all its derivatives will vanish identically. The function $\eta_{j, x}$ is Borel measurable because it is continuous on the Borel set $B_{j}(x)$.

\subsection{An aside on functions of finite type}

An important notion tied to the estimation of oscillatory integrals is that of functions of finite type. While there are many variations and generalizations of this notion appearing in the literature, at its core, a function of finite type is one which is nearly polynomial. The principal benefit of restricting attention to these functions is that they satisfy an inequality of the form

$$
\sup _{|\omega|<1}\left|\partial^{\alpha} f(\omega)\right| \leq C_{\alpha, f} \sup _{|\omega|<1}|f(\omega)|
$$

for some constant $C_{\alpha, f}$ which is nearly independent of $f$ (e.g., in terms of its degree when $f$ is a polynomial, or, more generally, in terms of the ratio of its $C^{m}$ norm to some lower bounds for the nonvanishing derivative, etc.). This inequality may be thought of as bounding the high frequency components of $f$ by the low frequencies (and so we have the heuristic that functions which are locally like polynomials are also locally like slowly varying complex exponential functions). A closely related problem is to determine the maximal $\epsilon$ such that

$$
\sup _{|\omega|<1}\left|\partial^{\alpha} f(\omega)\right| \leq \epsilon^{-|\alpha|} \sup _{|\omega|<1}|f(\omega)|
$$

holds for a given $f$ and a certain range of derivatives $\alpha$. The reader should note that as $f$ becomes more oscillatory, one expects the maximal $\epsilon$ to become smaller, and there is a natural limit forbidding $\epsilon$ from ever being larger than roughly unit size in any nontrivial situation (since, for example, one must have $\epsilon \leq 1 / 2$ when $f$ is merely a linear function vanishing at $\omega=0$ ). Theorem 2.1 gives a sharp answer to this question of determining the optimal $\epsilon$ up to a universal constant depending only on dimension and the degree of smoothness of $f$. It gives a quantitative analogue of the finite-type condition which is useful in the proof of Theorem 1.1 and is hopefully interesting in its own right. The proof is accomplished by adapting the usual real variable Littlewood-Paley methods to the setting of the unit ball. 
Theorem 2.1. Suppose $f \in C^{m}(\mathbb{B})$ satisfies the inequality

$$
\sup _{\substack{|\omega|<1 \\|\alpha|=m}} \epsilon^{|\alpha|}\left|\partial^{\alpha} f(\omega)\right| \leq \sup _{\substack{|\omega|<1 \\ \ell \leq \leq|<|<m}} \epsilon^{|\alpha|}\left|\partial^{\alpha} f(\omega)\right|
$$

for some positive $\epsilon \leq 1$ and some integer $\ell<m$. Then there is an implicit constant depending only on the dimension and $m$ such that

$$
\sup _{|\omega|<1}\left|\partial^{\alpha} f(\omega)\right| \lesssim \epsilon^{-|\alpha|} \sup _{\substack{|\omega|<1 \\|\beta|=\ell}} \epsilon^{|\beta|}\left|\partial^{\beta} f(\omega)\right|
$$

for all multi-indices $\ell \leq|\alpha| \leq m$.

Note that (2.5) clearly implies (2.4) for some $\epsilon^{\prime}$ differing from $\epsilon$ by a uniform constant. As such we essentially have a characterization of (2.5). The theorem, however, is far from tautological, since (2.4) is easily verified while (2.5) is not. In particular (2.4) is immediately true with $\epsilon=1$ when $f$ is a polynomial of degree at most $m-1$. Likewise it is easy to see that an acceptable $\epsilon$ satisfying $(2.4)$ and proportional to ratio $\|f\|_{C^{m-1}(\mathbb{B})} /\|f\|_{C^{m}(\mathbb{B})}$ may be given (which is how the theorem is typically applied to functions of finite type). A third example, relevant to the Carnot-Carathéodory geometry, is given after the proof. Finally, we note that, although the $\epsilon$ dependence in Theorem 2.1 can be removed by the transformation $\omega^{\prime}:=\epsilon^{-1} \omega$, the hypotheses of the theorem are not actually scale invariant. It is not necessarily the case, for example, that for fixed $f$

$$
\sup _{\substack{\left|\omega^{\prime}\right|<R \\|\alpha|=m}}\left|\partial^{\alpha} f\left(\omega^{\prime}\right)\right| \leq \sup _{\substack{\left|\omega^{\prime}\right|<R \\ \ell \leq|\alpha|<m}}\left|\partial^{\alpha} f\left(\omega^{\prime}\right)\right|
$$

can be made true for any value of $R$, large or small, while (2.4) will clearly be satisfiable for small $\epsilon$ for any fixed $f$ (since the only possible obstruction occurs when the right-hand side is identically zero, but then the left-hand side will be identically zero as well).

Proof of Theorem 2.1. Let $\varphi$ be a smooth function compactly supported in $\mathbb{B}$. We suppose that $\varphi$ is even and satisfies the moment conditions

$$
\int_{\mathbb{B}} \varphi(x) d x=1 \quad \text { and } \quad \int_{\mathbb{B}} x^{\alpha} \varphi(x) d x=0 \quad \text { when } 1 \leq|\alpha| \leq m-1 .
$$

Using this $\varphi$, we consider the following operator for each nonnegative integer $j$ :

$$
P_{j} f(x, h):=2^{d j} \int f(x-z) \sum_{k=0}^{m} 2^{k j} \frac{\left((h \cdot \nabla)^{k} \varphi\right)\left(2^{j} z\right)}{k !} d z .
$$

This is well defined for bounded, continuous functions on the ball provided that $|x| \leq 1-2^{-j}$. Note that the dependence on $h$ is polynomial. In particular, with $x$ fixed, the $h$ dependence is exactly the degree $m$ Taylor polynomial of $P_{j} f(x+h, 0)$ at $h=0$, so $P_{j} f(x, h)=f(x+h)$ when $f$ is any polynomial of degree at most $m$. 
We may therefore reasonably think of $P_{j} f(x, h)$ as an analogue of the LittlewoodPaley projection of $f$ onto frequencies $2^{j}$ and below. Just as with Littlewood-Paley projections, we have uniform estimates

$$
\sup _{|x| \leq 1-2^{-j}}\left|\partial_{h}^{\beta} P_{j} f(x, h)\right| \lesssim 2^{j|\beta|}\left(1+\left(2^{j}|h|\right)^{m-|\beta|}\right) \sup _{|\omega|<1}|f(\omega)|
$$

with an implied constant that is independent of $f, h$, and $j$ (but may depend on the multi-indices, $m$, and the dimension).

If $f \in C^{\ell}(\mathbb{B})$ for some $\ell \leq m$, we may integrate by parts to conclude

$$
\begin{aligned}
P_{j} f(x, h)= & 2^{d j} \int \varphi\left(2^{j}(x-z)\right) \sum_{k=0}^{\ell-1} \frac{\left((h \cdot \nabla)^{k} f\right)(z)}{k !} d z \\
& +2^{d j} \int\left((h \cdot \nabla)^{\ell} f\right)(x-z) \sum_{k=0}^{m-\ell} 2^{k j} \frac{\left((h \cdot \nabla)^{k} \varphi\right)\left(2^{j} z\right)}{(k+\ell) !} d z .
\end{aligned}
$$

In particular, if $|\beta| \geq \ell$, then the partial derivative $\partial_{h}^{\beta}$ kills the first term on the right-hand side of (2.7). Thus we have a slight improvement of (2.6):

$$
\sup _{\substack{|x| \leq 1-2^{-j} \\|h| \leq 2^{-j}}}\left|\partial_{h}^{\beta} P_{j} f(x, h)\right| \lesssim 2^{j(|\beta|-\ell)} \sup _{\substack{|\omega|<1 \\|\gamma|=\ell}}\left|\partial^{\gamma} f(\omega)\right| .
$$

Returning to (2.7), changing variables in the first integral on the right-hand side gives

$$
\int \varphi\left(2^{j}(x-z)\right) \sum_{k=0}^{\ell-1} \frac{\left((h \cdot \nabla)^{k} f\right)(z)}{k !} d z=\int \varphi\left(2^{j} z\right) \sum_{k=0}^{\ell-1} \frac{\left((h \cdot \nabla)^{k} f\right)(x-z)}{k !} d z .
$$

By Taylor's Theorem, we have the inequalities

$$
\begin{gathered}
\left|\sum_{k=0}^{\ell-1} \frac{\left((h \cdot \nabla)^{k} f\right)(x-z)}{k !}-f(x-z+h)\right| \leq \sup _{|\omega|<1} \frac{\left|(h \cdot \nabla)^{\ell} f(\omega)\right|}{\ell !}, \\
\left|\sum_{k=0}^{\ell-1} \frac{\left((-z \cdot \nabla)^{k} f\right)(x+h)}{k !}-f(x-z+h)\right| \leq \sup _{|\omega|<1} \frac{\left|(z \cdot \nabla)^{\ell} f(\omega)\right|}{\ell !},
\end{gathered}
$$

provided that $x-z$ and $x-z+h$ belong to the unit ball in the first case and $x+h$ and $x-z+h$ belong to the ball in the second case. In particular, the second inequality contains a polynomial in $z$ of degree less than $m$; if multiplied by $\varphi\left(2^{j} z\right)$ and integrated in $z$, all terms but the constant term would cancel. We conclude from these estimates and (2.7) that the quantity $\left|P_{j} f(x, h)-f(x+h)\right|$ is dominated by

$$
\begin{aligned}
& 2^{d j} \int\left|\varphi\left(2^{j} z\right)\right|\left[\sup _{|\omega|<1} \frac{\left|(h \cdot \nabla)^{\ell} f(\omega)\right|}{\ell !}+\sup _{|\omega|<1} \frac{\left|(z \cdot \nabla)^{\ell} f(\omega)\right|}{\ell !}\right] d z \\
& \quad+2^{d j} \int\left[\sup _{|\omega|<1} \frac{\left|(h \cdot \nabla)^{\ell} f(\omega)\right|}{\ell !}\right] \sum_{k=0}^{m-\ell} 2^{k j}\left|\frac{\left((h \cdot \nabla)^{k} \varphi\right)\left(2^{j} z\right)}{(k+\ell) !}\right| d z .
\end{aligned}
$$


From this it is easy to see that there must be an implicit constant depending only on $\varphi$, the dimension, and $m$, so that $|x| \leq 1-2^{-j}$ and $|x+h|<1$ imply

$$
\left|P_{j} f(x, h)-f(x+h)\right| \lesssim 2^{-j \ell}\left(1+\left(2^{j}|h|\right)^{m}\right) \sup _{\substack{|\omega|<1 \\|\beta|=\ell}}\left|\partial^{\beta} f(\omega)\right| .
$$

It should also be noted that the inequality (2.9) will also hold trivially when $\ell=0$ by virtue of (2.6).

For fixed $f$, let $g_{j}^{x}(y):=P_{j} f(x, y-x)$. Because the dependence of $g_{j}^{x}(y)$ on $y$ is polynomial of degree at most $m$, we have that

$$
P_{j-1} g_{j}^{x}(x+\delta, h-\delta)=g^{x}(x+h)=P_{j} f(x, h) .
$$

Comparison with $P_{j-1} f(x+\delta, h+\delta)$ yields

$$
P_{j} f(x, h)=P_{j-1} f(x+\delta, h-\delta)+P_{j-1}\left(g_{j}^{x}-f\right)(x+\delta, h-\delta)
$$

provided $|x+\delta| \leq 1-2^{-j+1}$ and $|x| \leq 1-2^{-j}$. Now every point in the ball of radius $1-2^{-j}$ is within distance $2^{-j}$ of a point in the ball of radius $1-2^{-j+1}$. Let $E_{j}$ be the set of pairs $(x, h)$ where $|x| \leq 1-2^{-j},|x+h|<1$, and $|h|<2^{-j+1}$; we have

$$
\begin{aligned}
\sup _{\substack{|x| \leq 1-2^{-j} \\
|h| \leq 2^{-j}}}\left|\partial_{h}^{\alpha} P_{j} f(x, h)\right|- & \sup _{\substack{|x| \leq 1-2^{-j+1} \\
|h| \leq 2^{-j+1}}}\left|\partial_{h}^{\alpha} P_{j-1} f(x, h)\right| \\
& \lesssim 2^{j|\alpha|} \sup _{(x, h) \in E_{j}}\left|P_{j} f(x, h)-f(x+h)\right|
\end{aligned}
$$

where the implicit constant comes from (2.6). In light of (2.8), summing $j$ from $N+1$ to infinity and using the fact that $\partial_{h}^{\alpha} P_{j} f(x, h)$ at $h=0$ tends to $\partial^{\alpha} f(x)$ as $j \rightarrow \infty$ when $f \in C^{m}(\mathbb{B})$ and $|\alpha| \leq m$ (shown by integration by parts as usual) gives

$$
\begin{aligned}
\sup _{|\omega|<1}\left|\partial^{\alpha} f(\omega)\right| \lesssim & 2^{(|\alpha|-\ell) N} \sup _{\substack{|\omega|<1 \\
|\beta|=\ell}}\left|\partial^{\beta} f(\omega)\right| \\
& +\sum_{j=N+1}^{\infty} 2^{|\alpha| j} \sup _{(x, y) \in E_{j}}\left|P_{j} f(x, y)-f(x+y)\right| .
\end{aligned}
$$

Now suppose that $f$ satisfies the inequality (2.4) for some $\epsilon \leq 1$. Choose $N$ so that $2^{N}=\epsilon^{-1} \delta^{-1}$ for some $\delta<1$ to be chosen momentarily. Applying the inequalities (2.9) (with $\ell$ replaced by $m$ ) and (2.4) to the sum on the right-hand side of (2.10) gives

$$
\begin{aligned}
\sup _{|\omega|<1}\left|\partial^{\alpha} f(\omega)\right| & \lesssim(\epsilon \delta)^{\ell-|\alpha|} \sup _{\substack{|\omega|<1 \\
|\beta|=\ell}}\left|\partial^{\beta} f(\omega)\right|+(\epsilon \delta)^{m-|\alpha|} \sup _{\substack{|\omega|<1 \\
|\beta|=m}}\left|\partial^{\beta} f(\omega)\right| \\
& \lesssim \epsilon^{-|\alpha|}\left[\begin{array}{c}
\left.\delta^{\ell-|\alpha|} \sup _{\substack{|\omega|<1 \\
|\beta|=\ell}} \epsilon^{|\beta|}\left|\partial^{\beta} f(\omega)\right|+\delta^{m-|\alpha|} \sup _{\substack{|\omega|<1 \\
|\beta|<m}} \epsilon^{|\beta|}\left|\partial^{\alpha} f(\omega)\right|\right]
\end{array}\right.
\end{aligned}
$$

(for $|\alpha|=\ell$ this inequality is trivially true). 
If we choose $\delta$ small enough that $\delta$ times the implicit constant is between $1 / 4$ and $1 / 2$ (this will always be possible since we can increase the magnitude of the implicit constant as necessary), we may take a supremum over all such $\alpha$ with $\ell \leq|\alpha|<m$ and conclude

$$
\sup _{\substack{|\omega|<1 \\ \ell \leq|\alpha|<m}} \epsilon^{|\alpha|}\left|\partial^{\alpha} f(\omega)\right| \lesssim \sup _{\substack{|\omega|<1 \\|\beta|=\ell}} \epsilon^{|\beta|}\left|\partial^{\beta} f(\omega)\right| .
$$

Combining this inequality with (2.4) itself handles the case of multi-indices $\alpha$ with $|\alpha|=m$.

We now return to the issue of establishing the hypothesis (2.4) in a manner relevant to Carnot-Carathéodory geometry. Suppose $Y_{1}, \ldots, Y_{d}$ are $C^{m}$ vector fields on $\mathbb{B}^{d}$. We will say that a function $f$ is of polynomial type with respect to $Y_{1}, \ldots, Y_{d}$ if

$$
Y_{i_{1}} \cdots Y_{i_{M}} f \equiv 0
$$

for some fixed $M$ and all choices $\left(i_{1}, \ldots, i_{M}\right) \in\{1, \ldots, d\}^{M}$. Any such function automatically satisfies (2.4) for some $\epsilon$ that depends only on the $C^{m}$ norms of the vector fields $Y_{i}$, the infimum of the absolute value of the $\operatorname{determinant} \operatorname{det}\left(Y_{1}, \ldots, Y_{d}\right)$, and the constant $M$. This is because we may write

$$
\frac{\partial}{\partial x_{j}}=\sum_{i=1}^{d} c_{j i}(x) Y_{i}
$$

for some functions $c_{i j} \in C^{m}(\mathbb{B})$ whose norms depend only on the norms of the $Y_{i}$ and the lower bound from the determinant; this fact is easily seen from Cramer's rule. In particular, we see that the mixed partial derivative $\partial^{\beta} f$ may be written as a $C^{m}$ linear combination of the derivatives $Y_{i_{1}} \cdots Y_{i_{k}}$ for $k \leq M$. Since these expressions vanish identically when $k=M$, we see that the order $M$ partial derivatives of $f$ are smooth linear combinations of lower order partial derivatives, and the coefficients do not depend on $f$.

\section{Proof of Theorem 1.1}

\subsection{Covering lemma}

The benefit of the smoothness assumptions is that they allow the passage from a Vitali or Besicovitch-type covering lemma to a smooth partition of unity whose derivatives are well controlled.

Lemma 3.1. Fix some positive integer $N$ and some open subset $E \subset \Omega_{0}$. Suppose $R: E \rightarrow \mathbb{Z}$ is a bounded function such that $B_{R(x)+1}(x)$ is well-defined for each $x \in E$ and

$$
B_{R(x)}(x) \cap B_{R\left(x^{\prime}\right)}\left(x^{\prime}\right) \neq \emptyset \quad \Longrightarrow \quad\left|R(x)-R\left(x^{\prime}\right)\right|<N .
$$

Then there is a special collection $G$ of points $x \in E$ and nonnegative functions $\eta_{x}$ for each $x \in G$ which has the following properties. First, $\eta_{x}$ is identically zero out- 
side $B_{R(x)}(x)$. Next, at every $y \in E$, there is a ball $B_{j}(y)$ for which there is at most a uniformly bounded number of points $x \in G$ at which $B_{R(x)}(x) \cap B_{j}(y)$ $\neq \emptyset$. The functions $\eta_{x}$ are uniformly smooth in the sense that $x \in G$ and $\| \eta_{x} \circ$ $\Phi_{R(x), x} \|_{C^{m}(\mathbb{B})} \lesssim 1$. Finally, $\sum_{x \in G} \eta_{x}=1$ on $E$.

Proof. Let $E_{j} \subset E$ be the set of points $x$ for which $R(x)=j$. For each $j$, let $G_{j} \subset E_{j}$ be any maximal collection of points $x$ such that $B_{j-2}(x) \cap B_{j-2}\left(x^{\prime}\right)=\emptyset$ for any two $x, x^{\prime} \in G_{j}$ (all these balls must exist by virtue of the compatibility condition). First we note that the union over all $x \in G_{j}$ of the balls $B_{j-1}(x)$ will cover $E_{j}$; for any $y \in E_{j}, B_{j-2}(y) \cap B_{j-2}(x) \neq \emptyset$ for some $x \in G_{j}$ (if not, $y$ itself could be added to $G_{j}$ to contradict maximality). The engulfing property guarantees that $B_{j-2}(y) \subset B_{j-1}(x)$. Next observe that the family of balls $B_{j}(x)$ for $x \in G_{j}$ is locally finite in the following sense: for any $x \in E$, let $S$ be the set of centers $x^{\prime} \in G_{j}$ such that $B_{j}(x) \cap B_{j}\left(x^{\prime}\right) \neq \emptyset$ (if $B_{j}(x)$ is not defined, the set $S$ will be trivial). This set $S$ must necessarily be finite with uniformly bounded cardinality. To see this, let $S_{1}$ be any maximal subset of $S$ comprising disjoint balls at scale $j-1$, i.e., $B_{j-1}\left(x^{\prime}\right) \cap B_{j-1}\left(x^{\prime \prime}\right)=\emptyset$ for any $x^{\prime}, x^{\prime \prime} \in S_{1}$. In general, let $S_{k}$ be a maximal subset of $S \backslash \bigcup_{l=1}^{k-1} S_{l}$ comprising disjoint balls at scale $j-1$. The weak doubling property dictates that $S_{C+2}$ is empty for some universal $C$, since maximality dictates that $x^{\prime} \in S_{C+1}$ implies that $B_{j-1}\left(x^{\prime}\right) \cap B_{j-1}\left(x_{k}\right) \neq \emptyset$ for $k=1, \ldots, C+1$ and some $x_{k} \in S_{k}$ (which cannot happen because the balls are all mutually disjoint at scale $j-2$ ). By weak doubling again, the number of points $x^{\prime} \in S_{k}$ at which $B_{j}(x) \cap B_{j}\left(x^{\prime}\right) \neq \emptyset$ is also at most $C$ for any fixed $k$. Thus the total number of indices in $S$ which produce balls at scale $j$ meeting $B_{j}(x)$ is at most $C(C+1)$. We may strengthen this result by taking a union over scales. For any point $x \in E$, the condition $\left|R(x)-R\left(x^{\prime}\right)\right|<N$ when $B_{R(x)}(x) \cap B_{R\left(x^{\prime}\right)}\left(x^{\prime}\right) \neq \emptyset$ implies that there are boundedly many indices $j^{\prime}$ for which $B_{R(x)}(x)$ intersects a ball $B_{R\left(x^{\prime}\right)}\left(x^{\prime}\right)$ with $x^{\prime} \in G_{j^{\prime}}$. If $j$ is any index which is bounded above by $j^{\prime}-1$ for each index $j^{\prime}$ identified above as well as bounded above by $R(x)-1$ (at least one such index, e.g., $j=R(x)-N$, is guaranteed to exist), then the number of points $x^{\prime} \in G_{j^{\prime}}$ for which $B_{j}(x) \cap B_{R\left(x^{\prime}\right)}\left(x^{\prime}\right) \neq \emptyset$ will be at most $C(C+1)$ (because we will have in particular that $B_{j}(x) \subset B_{R\left(x^{\prime}\right)}(x)$ by engulfing). Uniform boundedness on the cardinality of the possible values of $j^{\prime}$ gives a uniform bound on the number of nontrivial intersections $B_{j}(x) \cap B_{R\left(x^{\prime}\right)}\left(x^{\prime}\right) \neq \emptyset$ when $x^{\prime}$ is allowed to range over all of $G:=\bigcup_{j} G_{j}$.

As explained in (2.3), there is a natural choice of a smooth function subordinate to $B_{j}(x)$ for each $x \in G_{j}$, namely the function $\eta_{j, x}$. Furthermore we have $\| \eta_{j, x} \circ$ $\Phi_{R(y), y} \|_{C^{m}(\mathbb{B})} \lesssim 1$ for any $y \in E$ simply because $\eta_{j, x} \circ \Phi_{R(y), y}$ will be identically zero unless $B_{R(x)}(x) \cap B_{R(y)}(y) \neq \emptyset$, in which case we already have the uniform bound $|R(x)-R(y)|<N$ on the indices $R(x)$ and $R(y)$ (which finishes the job when combined with (2.3)). Now by the distributive law we have

$$
1=\prod_{x \in G}\left[\left(1-\eta_{R(x), x}\right)+\eta_{R(x), x}\right]=\sum_{\substack{S \subset G \\ \# S<\infty}}\left(\prod_{x \in S} \eta_{R(x), x}\right)\left(\prod_{y \in G \backslash S}\left(1-\eta_{R(y), y}\right)\right),
$$

since on any ball $B_{R(z)-N}(z)$ with $z \in E$ all but boundedly many choices of $x$ will 
have $\left(1-\eta_{R(x), x}\right)$ which is identically one on this ball since $\eta_{R(x), x}$ is identically zero.

Given a finite subset $S \subset G$, let $M(S)$ be the subset of $S$ drawn from the $G_{j}$ 's with maximal indices; specifically, for each $x \in S, x$ belongs to $G_{j}$ for a unique index $j$. We will take $x \in M(S)$ if and only if $S \cap G_{k}=\emptyset$ for all $k>j$. We will say that two finite subsets $S$ and $S^{\prime}$ are equivalent when $M(S)=M\left(S^{\prime}\right)$. On any equivalence class $\mathcal{S}, M(\mathcal{S})$ is well defined (since $M(S)$ is constant for all representatives $S$ ). If we call the collection of equivalence classes $\mathcal{E}$, we have

$$
1=\prod_{x \in G}\left(1-\eta_{R(x), x}\right)+\sum_{\mathcal{S} \in \mathcal{E} \backslash\{\emptyset\}} \sum_{S \in \mathcal{S}}\left(\prod_{x \in S} \eta_{R(x), x}\right)\left(\prod_{y \in G \backslash S}\left(1-\eta_{R(y), y}\right)\right)
$$

(where we identify $\emptyset \in \mathcal{E}$ with the equivalence class of the empty set). Since $\eta_{j, x}$ is identically one on $B_{j-1}(x)$ and the union of the balls $B_{R(x)-1}(x)$ over $x \in G$ covers $E$, the first product on the right-hand side will be identically zero on $E$.

For a fixed equivalence class $\mathcal{S}$, let $I_{\mathcal{S}}^{0}$ be the indices $j$ such that $G_{j} \cap M(\mathcal{S}) \neq \emptyset$, and let $I_{\mathcal{S}}^{-}$be the indices $j$ such that $j<k$ for some $k \in I_{\mathcal{S}}^{0}$. By definition, the representatives $S$ of the equivalence class $\mathcal{S}$ are given by the union of $M(\mathcal{S})$ with any fixed subset of $\bigcup_{j \in I_{\mathcal{S}}^{-}} G_{j}$. In particular, if $I_{\mathcal{S}}^{+}$is the complement of $I_{\mathcal{S}}^{0} \cup I_{\mathcal{S}}^{-}$, then every representative $S$ has $S \cap G_{j}=\emptyset$ when $j \in I_{\mathcal{S}}^{+}$. Consequently, the distributive law guarantees that

$$
\begin{aligned}
\sum_{S \in \mathcal{S}}\left(\prod_{x \in S} \eta_{R(x), x}\right)\left(\prod_{y \in G \backslash S}\left(1-\eta_{R(y), y}\right)\right) & \\
& =\left(\prod_{x \in M(\mathcal{S})} \eta_{R(x), x}\right)\left(\prod_{y \in G_{\mathcal{S}}^{+} \backslash M(\mathcal{S})}\left(1-\eta_{R(y), y}\right)\right)
\end{aligned}
$$

if we define $G_{\mathcal{S}}^{0}:=\bigcup_{j \in I_{\mathcal{S}}^{0}} G_{j}$ and $G_{\mathcal{S}}^{+}:=\bigcup_{j \in I_{\mathcal{S}}^{+}} G_{j} \cup \bigcup_{j \in I_{\mathcal{S}}^{0}} G_{j}$. Moreover, $B_{R(x)}(x) \cap$ $B_{R(y)}(y)=\emptyset$ when $|R(x)-R(y)| \geq N$, so the above formula remains true when $G_{\mathcal{S}}^{+}$ is replaced by the (substantially smaller) union of those $G_{j}$ for which $j \in I_{\mathcal{S}}^{+} \cup I_{\mathcal{S}}^{0}$ and $|j-k|<N$ for all $k \in I_{\mathcal{S}}^{0}$. Since this set has uniformly bounded cardinality, we may conclude that, for any $z \in E$, there is some ball $B_{j^{\prime}}(z)$ on which the set of equivalence classes $\mathcal{S}$ giving rise to a nontrivial (i.e., not identically zero) product has uniformly bounded cardinality. This coupled with smooth comparability guarantees that the composition of any such product with any $\Phi_{R(z), z}$ will have uniformly bounded norm in $C^{m}(\mathbb{B})$ (since at each point of the ball the function is locally a product of bounded cardinality, and the definition of $R$ implies that each ball appearing in the product will have index uniformly near $R(z)$ if the product is not simply identically zero). Finally, if we set

$$
\eta_{x}:=\sum_{\mathcal{S}: x \in M(\mathcal{S})} \frac{1}{\# M(\mathcal{S})}\left(\prod_{z \in M(\mathcal{S})} \eta_{R(z), z}\right)\left(\prod_{y \in G_{\mathcal{S}}^{+} \backslash M(\mathcal{S})}\left(1-\eta_{R(y), y}\right)\right)
$$

(which again is well defined, since for fixed $x$ only boundedly many choices of $\mathcal{S}$ 
will give a sum which is not identically zero on $\left.B_{R(x)}(x)\right)$, we have that

$$
1=\sum_{x \in G} \eta_{x}
$$

on $E$ and $\left\|\eta_{x} \circ \Phi_{R(x), x}\right\|_{C^{m}(\mathbb{B})}$ is uniformly bounded. That $\eta_{x}$ is supported on $B_{R(x)}(x)$ follows because the same is true of $\eta_{R(x), x}$.

It is important to note that the set $G$ constructed in the lemma is not necessarily countable and so we have not technically constructed a partition of unity in the usual sense. However, we have that $G \cap L$ is countable for any leaf $L$. This is because the sets $E \cap B_{R(x)}(x)$ are contained and open in $L$ for each $x$. Moreover, no point $y \in L$ is contained in more than boundedly many of these sets $E \cap B_{R(x)}(x)$ for $x \in E$. Since $L$ has a countable dense subset, the pigeonhole principle forces that there can be only countably many $x$ for which $E \cap B_{R(x)}(x) \cap L$ is nonempty for any particular leaf $L$ (as $x$ ranges over all of $G$ ). In the event that the function $R$ satisfies the condition (1.7) from the statement of Theorem 1.1, it is easy to see that

$$
\sum_{x \in G \cap L} \psi_{x}=\chi_{L \cap E}
$$

(where $\chi$ represents the characteristic function) for the simple reason that the complement of $E$ will be the set where $d f$ vanishes, and (1.7) guarantees that $B_{R(x)}(x)$ does not contain any such points whenever $x \in E$. By the factorization property of $\mu$, then, we have that

$$
\int_{\Omega} e^{i f} \psi d \mu=\int_{\Omega \backslash E} e^{i f} \psi d \mu+\int_{\mathcal{F}}\left(\sum_{x \in G \cap L} \int_{B_{R(x)}(x)} e^{i f} \psi \eta_{x} d \mu_{L}\right) d \mu_{\mathcal{F}}(L)
$$

(the assumption that $\psi$ is bounded and supported on a set of finite measure in $\Omega_{0}$ guarantees that we trivially have dominated convergence on almost every leaf $L$ ).

\subsection{Integral estimates and conclusion}

We have thus successfully reduced the problem to the very classical one of a scalar oscillatory integral on a Euclidean ball. The main result we will use in this context is contained in the following lemma.

Lemma 3.2. Suppose $f \in C^{m}(\mathbb{B})$ has nonvanishing gradient. Then for each $k=1, \ldots, m-1$, there exist functions $F_{\beta}$ for all multiindices $|\beta| \leq k$ such that

$$
\int_{\mathbb{B}} e^{i f} \psi d x=\int_{\mathbb{B}} e^{i f}\left[\sum_{|\beta| \leq k} \partial^{\beta} \psi(x) F_{\beta}(x)\right] d x
$$

for any compactly supported $\psi \in C^{m}(\mathbb{B})$. The functions $F_{\beta}$ satisfy the inequalities

$$
\left|F_{\beta}(x)\right| \lesssim \frac{(\omega(x))^{|\beta|}}{(\omega(x)|\nabla f(x)|)^{k}}
$$


for any nonnegative function $\omega$ satisfying

$$
\frac{1}{\omega(x)} \geq \sup _{2 \leq|\gamma| \leq k+1}\left(\frac{\left|\partial^{\gamma} f(x)\right|}{|\nabla f(x)|}\right)^{1 /(|\gamma|-1)} .
$$

The implicit constant in (3.3) depend only on the dimension and $m$.

Proof. The proof is by the time-honored method of integration by parts. Suppose that $u: \mathbb{R}^{d} \rightarrow \mathbb{C}$ is smooth and homogeneous of degree $a$. We integrate by parts as follows:

$$
\begin{aligned}
\int_{\mathbb{B}} e^{i f(x)} \partial^{\beta} \psi(x) & u(\nabla f(x)) \prod_{j=1}^{n} \partial^{\gamma_{j}} f(x) d x \\
& =\int_{\mathbb{B}} \frac{\nabla f(x)}{i|\nabla f(x)|^{2}} \cdot \nabla\left(e^{i f(x)}\right) \partial^{\beta} \psi(x) u(\nabla f(x)) \prod_{j=1}^{n} \partial^{\gamma_{j}} f(x) d x \\
& =i \int_{\mathbb{B}} e^{i f} \nabla \cdot\left(\partial^{\beta} \psi(x) \frac{u(\nabla f(x)) \nabla f(x)}{|\nabla f(x)|^{2}} \prod_{j=1}^{n} \partial^{\gamma_{j}} f(x)\right) d x .
\end{aligned}
$$

Now there are three cases to consider. If the derivatives present in the divergence fall on $\partial^{\beta} \psi$, then we may write the resulting terms as

$$
i \int_{\mathbb{B}} e^{i f} \sum_{\ell=1}^{d} \partial^{\beta+e_{\ell}} \psi(x) u_{\ell}(\nabla f(x)) \prod_{j=1}^{n} \partial^{\gamma_{j}} f(x)
$$

where $e_{\ell}$ is the multi-index corresponding to differentiation with respect to $x_{\ell}$ and $u_{\ell}(y):=u(y) y_{\ell}|y|^{-2}$, which will be smooth and homogeneous of degree $\alpha-1$. When the divergence falls on the $\nabla f$ terms, we get

$$
i \int_{\mathbb{B}} e^{i f} \partial^{\beta} \psi(x) \sum_{\ell=1}^{d} \sum_{k=1}^{d}\left(\partial_{k} u_{\ell}\right)(\nabla f(x)) \partial_{x_{\ell} x_{k}}^{2} f(x) \prod_{j=1}^{n} \partial^{\gamma_{j}} f(x) d x .
$$

When this is expanded, we find that each term remains of the same form with $u$ being replaced by $\partial_{k} u_{\ell}$ (which is smooth and homogeneous of degree $a-2$ ) and the cardinality of the product of higher derivatives increasing by one while the total number of derivatives present in the product increases by two. Finally, if the divergence falls on one of the higher derivatives of $f$, the only effect is to increase the order of differentiation on that term by 1 . By induction, we conclude that

$$
\int_{\mathbb{B}} e^{i f} \psi d x=\sum_{|\beta| \leq k} \int e^{i f} \partial^{\beta} \psi F_{\beta} d x
$$

as desired (in the base case, $u(\nabla f) \equiv 1$ is homogeneous of degree 0 ), where each $F_{\beta}$ is a linear combination (with universal coefficients depending only on the dimension, $k$, and $\beta$ ) of terms of the form

$$
u(\nabla f(x)) \prod_{j=1}^{n} \partial^{\gamma_{j}} f(x) .
$$


The index $n$ can be taken less than or equal to $k$ (the case equalling zero meaning no higher derivatives are present). Here $u$ will be smooth and homogeneous of degree no greater than $-k$. More precisely, an analysis of the three cases above yields that

$$
|\beta|+\operatorname{deg} u+\sum_{j=1}^{n}\left|\gamma_{j}\right|=0, \quad \operatorname{deg} u+n=-k, \quad \text { and } \quad k \leq|\beta|+\sum_{j=1}^{n}\left|\gamma_{j}\right| \leq 2 k .
$$

It is equally elementary to see that $\left|\gamma_{j}\right|$ can be at most $k+1$ for any $k$. In particular, given the definition of $\omega(x)$, we have that

$$
\prod_{j=1}^{n}\left|\partial^{\gamma_{j}} f(x)\right| \leq \prod_{j=1}^{n}|\nabla f(x)|(\omega(x))^{-\left|\gamma_{j}\right|+1}=(\omega(x)|\nabla f(x)|)^{n}(\omega(x))^{-\sum_{j=1}^{n}\left|\gamma_{j}\right|} .
$$

If we set $s=\sum_{j=1}^{d}\left|\gamma_{j}\right|$, then we can conclude that

$$
\begin{aligned}
\left|F_{\beta}(x)\right| & \lesssim \max _{s+|\beta|=k, \ldots, 2 k}|\nabla f(x)|^{-s-|\beta|}(\omega(x)|\nabla f(x)|)^{-k+|\beta|+s}(\omega(x))^{-s} \\
& \lesssim \frac{(\omega(x))^{|\beta|}}{(\omega(x)|\nabla f(x)|)^{k}},
\end{aligned}
$$

which finishes the proof.

Now we return to the expression (3.1) and apply (3.2). Specifically we have

$$
\int_{B_{R(x)}(x)} e^{i f} \psi \eta_{x} d \mu_{L}=\mu_{L}\left(B_{R(x)}(x)\right) \int_{\mathbb{B}} e^{i \tilde{f}(t)} \tilde{\psi}(t) d t
$$

where

$$
\begin{aligned}
\tilde{f}(t) & :=f \circ \Phi_{R(x), x}(t), \\
\tilde{\psi}(t) & :=\psi \circ \Phi_{R(x), x}(t) \eta_{x} \circ \Phi_{R(x), x}(t) J_{R(x), x}(t) .
\end{aligned}
$$

By design, the product $\eta_{x} \circ \Phi_{R(x), x}(t) J_{R(x), x}(t)$ has uniformly bounded norm in $C^{m}(\mathbb{B})$ and so can (in essence) be neglected.

Let $C$ be a constant to be chosen momentarily. If $\left|d_{y} f\right|_{R(y)} \leq C \epsilon^{-1}$ for all points $y \in B_{R(x)}(x)$, then observe that we have the trivial identity

$$
\int_{\mathbb{B}} e^{i \tilde{f}(t)} \tilde{\psi}(t) d t=\int_{\mathbb{B}} e^{i \tilde{f}(t)} \tilde{\psi}(t) d t
$$

and the trivial inequality

$$
|\tilde{\psi}(t)| \lesssim \frac{\left|\psi \circ \Phi_{R(x), x}(t)\right|}{\left(1+\epsilon\left|d_{y} f\right|_{R(y)}\right)^{m-1}}
$$

for any $y \in B_{R(x)}(x)$ (with an implicit constant depending on $C$ ). 
If $\left|d_{y} f\right|_{R(y)} \geq C \epsilon^{-1}$ at some point $y \in B_{R(x)}(x)$, then the assumption (1.7) implies a uniform bound from below at every point in the ball when $C$ is chosen sufficiently large relative to the implicit constant in (1.7). In this case we will apply Lemma 3.2 to the oscillatory integral. Observe that when $y=\Phi_{R(x), x}(t)$, we have

$$
\sup _{1 \leq|\gamma| \leq k} \frac{\left|\partial^{\gamma} \tilde{f}(t)\right|}{|\nabla f(t)|} \approx \frac{\left|d_{y}^{k} f\right|_{B_{R(x)}(x)}}{\left|d_{y} f\right|_{B_{R(x)}(x)}}
$$

(with universal constants depending only on dimension). Since $y \in B_{R(x)}(x)$ and consequently $|R(x)-R(y)|<N$, by (2.2) we have that

$$
\left|d_{y}^{k} f\right|_{B_{R(x)}(x)} \approx\left|d_{y}^{k} f\right|_{B_{R(y)}(y)}=\left|d_{y}^{k} f\right|_{R(y)}
$$

uniformly for any $k=1, \ldots, m$ (with implied but uninteresting dependence on $N$ as well as the usual constants). By (1.6) and Theorem 2.1, now, we can conclude that the ratio (3.6) is bounded above, uniformly in $y$, by $\epsilon^{-k}$ and thus the function $\omega$ from Lemma 3.2 can be taken equal to some uniform constant times $\epsilon$. We can conclude that

$$
\int_{\mathbb{B}} e^{i \tilde{f}(t)} \tilde{\psi}(t) d t=\int_{\mathbb{B}} e^{i \tilde{f}(t)} \tilde{\psi}_{m}(t) d t
$$

and

$$
\left|\tilde{\psi}_{m}(t)\right| \lesssim \frac{\sum_{k=0}^{m-1} \epsilon^{k}\left|d_{y}^{k} \psi\right|_{R(y)}}{\left(1+\epsilon\left|d_{y} f\right|\right)^{m-1}}
$$

uniformly when $\Phi_{R(x), x}(y)=t$ (by virtue of (3.3) and the fact that $\left|d_{y} f\right| \approx 1+\left|d_{y} f\right|$ on this particular ball).

Next take either (3.5) or (3.7) and transform back to the measure $\mu_{L}$; in the case of (3.5), we define the function $\psi_{x}$ by $\psi_{x}:=\psi \eta_{x}$, and in the case of (3.7) we take

$$
\psi_{x}:=\left(\frac{\tilde{\psi}_{m}}{J_{R(x), x}}\right) \circ \Phi_{R(x), x}^{-1} .
$$

Since $J_{R(x), x}^{-1}$ is uniformly bounded above, we may conclude that (in both cases) we have an equality

$$
\int e^{i f} \psi \eta_{x} d \mu_{L}=\int e^{i f} \psi_{x} d \mu_{L}
$$

where $\psi_{x}$ is zero outside $B_{R(x)}(x)$ and

$$
\left|\psi_{x}(y)\right| \lesssim \frac{\sum_{k=0}^{m-1} \epsilon^{k}\left|d_{y}^{k} \psi\right|_{R(y)}}{\left(1+\epsilon\left|d_{y} f\right|\right)^{m-1}}
$$

when $y \in B_{R(x)}(x)$ (with a constant uniform in $x$ and $y$ ). We now invert the interchange of summation and integration in (3.1). Because the balls chosen in Lemma 3.1 were locally finite on $E$, the uniform bound just established for $\left|\psi_{x}(y)\right|$ continues to hold when summed over $x \in G \cap L$ on any choice of leaf $L$. This establishes Theorem 1.1, the main theorem and, in particular, the desired inequal- 
ity (1.8). We also note that everything here is Borel measurable so the inversion of (3.1) is justified. This concludes the proof of Theorem 1.1.

It is possible to to remove the assumption that the Jacobian from (1.4) is bounded below if one is willing to pay a price in terms of the amplitude $\psi$. Instead of (3.8), the equalities

$$
\begin{aligned}
\int_{\mathbb{B}} e^{i \tilde{f}(t)} \tilde{\psi}_{m}(t) d t & =\int_{\mathbb{B}} J_{R(x), x}(s) d s \int_{\mathbb{B}} e^{i \tilde{f}(t)} \tilde{\psi}_{m}(t) d t \\
& =\int_{\mathbb{B}} e^{i \tilde{f}(s)}\left[\int_{\mathbb{B}} e^{i(\tilde{f}(t)-\tilde{f}(s))} \tilde{\psi}_{m}(t) d t\right] J_{R(x), x}(s) d s
\end{aligned}
$$

mean that one could alternately define

$$
\psi_{x}(\cdot):=\int_{\mathbb{B}} e^{i\left(\tilde{f}(t)-\tilde{f} \circ \Phi_{R(x), x}^{-1}(\cdot)\right)} \tilde{\psi}_{m}(t) d t .
$$

One would no longer need the Jacobian to be bounded below, but one would pay for it by selecting an amplitude $\psi_{x}$ (and hence an amplitude $\psi_{m}$ in Theorem 1.1) which is only bounded above by a sort of maximal function of the derivatives of the original phase as opposed to a simpler pointwise supremum of those derivatives.

\section{Applications and extensions}

\subsection{A canonical construction of scale function $R(x)$}

The selection of the scale function $R$ may be, in the general case when scales are understood in the multiparameter sense, more of an art than a science. However, in the single scale case (corresponding to scales parametrized by the integers $\mathbb{Z}$ ), it is relatively easy to see that there is always, in some sense, a "best" choice of $R$. Fix an $\epsilon$ and some implicit constants, and let $\mathcal{I}$ be the subcollection of balls $B_{j}(x)$, as $(j, x)$ ranges over pairs $\mathbb{Z} \times \Omega$, for which

$$
\left|d_{x}^{m}\right|_{j} \lesssim \sum_{k=1}^{m-1} \epsilon^{k-m}\left|d_{x}^{k} f\right|_{j} \text { and } \sup _{y \in B_{j}(x)}\left|d_{y} f\right|_{j-1} \lesssim \inf _{y \in B_{j}(x)}\left|d_{y}\right|_{j-1} .
$$

Let $\mathcal{I}$ denote this subset of $\mathbb{Z} \times \Omega$. We will define $R_{\mathcal{I}}(x)$ to be the supremum over those indices $j$ such that $B_{j+1}(x)$ exists and $B_{j^{\prime}}\left(x^{\prime}\right) \in I$ for any ball $B_{j^{\prime}}\left(x^{\prime}\right) \subset$ $B_{j+1}(x)$ with $j^{\prime} \leq j$.

The claim is that this mapping $R_{\mathcal{I}}$ satisfies the necessary regularity condition (1.5). Specifically, let $E$ be the set of points $x \in \Omega$ at which $R_{\mathcal{I}}(x)$ is well defined and finite. For any $x, x^{\prime} \in E$, suppose that $B_{R_{\mathcal{I}}(x)}(x) \cap B_{R_{\mathcal{I}}\left(x^{\prime}\right)}\left(x^{\prime}\right) \neq \emptyset$. By compatibility and nesting, $B_{R_{\mathcal{I}}(x)}\left(x^{\prime}\right)$ exists and is contained in $B_{R_{\mathcal{I}}+1}(x)$, and by the definition of $R_{\mathcal{I}}(x)$, it must therefore be the case that all balls of scale at most $R_{\mathcal{I}}(x)-1$ which are contained in $B_{R_{\mathcal{I}}(x)}\left(x^{\prime}\right)$ must also belong to $I$. We thus conclude that $R_{\mathcal{I}}\left(x^{\prime}\right) \geq R_{\mathcal{I}}(x)-1$. By symmetry we have $\left|R_{\mathcal{I}}\left(x^{\prime}\right)-R_{\mathcal{I}}(x)\right| \leq 2$. Because $B_{R_{\mathcal{I}}(x)}(x)$ belongs to $\mathcal{I}$, it is also an immediate consequence that both (1.6) and (1.7) hold. Thus we have established in a very explicit way that the scale 
function can simply be taken to measure the largest scale on which (1.6) and (1.7) hold. If the phase $f$ is finite type in the sense mentioned at the end of section 2.2 (which will happen when $f$ is polynomial or, in the Carnot-Carathéodory context, when $f$ is annihilated by applying any sufficiently long sequence of distinguished vector fields), then the scale function will simply measure the largest scale on which the magnitude of the derivative is constant.

\subsection{Quantitative results for convex phases}

In this section we establish several results relating Theorem 1.1 to the earlier theorem of Bruna, Nagel, and Wainger. Specifically we consider the question of establishing uniform radial tameness for an appropriate phase or phases and then give the proof of Corollary 1.2. We begin with two propositions which establish uniform radial tameness, first for polynomial convex phases and then for convex phases of finite type (in the sense of Bruna, Nagel, and Wainger).

Proposition 4.1. Suppose $f$ is a convex function on a convex open set $\Omega \subset \mathbb{R}^{d}$ containing the origin and that $f$ and its gradient vanish at the origin. If $f$ is a polynomial then it is uniformly radially tame to order $m$ with a constant depending only on the degree and $m$.

Proof. It suffices to restrict attention to a single ray emanating from the origin. Suppose $f(t)$ is a convex polynomial on $[0, T]$ with $f(0)=f^{\prime}(0)=0$. Rescaling $(2.5)$ to the interval $\left(0, t_{0}\right)$, we have that

$$
t_{0}^{k} \sup _{0<t<t_{0}}\left|f^{(k)}(t)\right| \lesssim \sup _{0<t<t_{0}}|f(t)|
$$

for any $k$, with an implicit constant depending only on $k$ and the degree of $f$. Convexity implies that, for all $t$,

$$
f^{\prime}(t) \geq t^{-1} f(t)
$$

so we conclude from (2.5) and monotonicity of $f$ that

$$
t_{0} f^{\prime}\left(t_{0}\right) \approx f\left(t_{0}\right)
$$

for all $t_{0} \in(0, T)$, and so we can additionally conclude that

$$
\left|f\left(t_{0}\right)\right|^{k-1}\left|f^{(k)}\left(t_{0}\right)\right| \lesssim\left|f^{\prime}\left(t_{0}\right)\right|^{k}
$$

for any $t_{0} \in(0, T)$ with an implicit constant depending only on the degree of $f$ and on $k$.

Proposition 4.2. Let $f$ be a smooth convex function on a convex open set $\Omega \subset \mathbb{R}^{d}$. Suppose that $f$ is finite type in the sense of Bruna, Nagel, and Wainger, namely, that every tangent line to $f$ has only finite order of contact. For $x_{0}$ belonging to any compact convex subset $\Omega^{\prime} \subset \Omega$, the convex functions

$$
f_{x_{0}}(x):=f(x)-f\left(x_{0}\right)-\left(x-x_{0}\right) \cdot \nabla f\left(x_{0}\right)
$$

are uniformly radially tame on $\Omega^{\prime}$ to any finite order $m$ with respect to the origin $x_{0}$, and the constant is bounded over all $x_{0} \in \Omega^{\prime}$. 
Proof. The finite type condition guarantees that for every point $x_{0}$ and unit vector $\omega$, there is a finite $k \geq 2$ such that

$$
\sum_{i=2}^{k}\left|(\omega \cdot \nabla)^{i} f\left(x_{0}\right)\right| \neq 0 .
$$

Since this sum (for fixed $k$ ) is a continuous function of $x_{0}$ and $\omega$, by compactness we can assume that there is a single $k$ such that

$$
\sum_{i=2}^{k} \mid\left(\omega \cdot \nabla^{i} f\left(x_{0}\right) \mid \geq C_{\Omega^{\prime}}>0\right.
$$

for any pair $\left(x_{0}, \omega\right) \in \Omega^{\prime} \times \mathbb{S}^{d-1}$. Now we can conclude that

$$
\sup _{1 \leq i \leq k} \sup _{0<t<1}\left|x-x_{0}\right|^{i}\left|\frac{d^{i}}{d t^{i}} f\left(x_{0}+t\left(x-x_{0}\right)\right)\right| \geq C_{\Omega^{\prime}}
$$

whenever $x_{0}$ and $x$ belong to $\Omega^{\prime}$. Now

$$
\begin{aligned}
\sup _{0<t<1}\left|x-x_{0}\right|^{m} & \left|\frac{d^{m}}{d t^{m}} f\left(x_{0}+t\left(x-x_{0}\right)\right)\right| \lesssim\|f\|_{C^{m}\left(\Omega^{\prime}\right)} \\
& \lesssim \frac{\|f\|_{C^{m}\left(\Omega^{\prime}\right)}}{C_{\Omega^{\prime}}} \sup _{1 \leq i \leq k} \sup _{0<t<1}\left|x-x_{0}\right|^{i}\left|\frac{d^{i}}{d t^{i}} f\left(x_{0}+t\left(x-x_{0}\right)\right)\right| .
\end{aligned}
$$

This inequality guarantees that $(2.4)$ will hold uniformly on $(0,1)$ for the functions $t \mapsto f\left(x_{0}+t\left(x-x_{0}\right)\right)-f\left(x_{0}\right)-t\left(x-x_{0}\right) \cdot \nabla f\left(x_{0}\right)$ with $x, x_{0} \in \Omega^{\prime}$ provided that $\epsilon$ is chosen to be a suitably small constant multiple of $\left|x-x_{0}\right|$. Thus we can conclude

$$
\sup _{0<t<1}\left|x-x_{0}\right|^{k}\left|\frac{d^{k}}{d t^{k}} f_{x_{0}}\left(x_{0}+t\left(x-x_{0}\right)\right)\right| \lesssim\left|f(x)-f\left(x_{0}\right)-\left(x-x_{0}\right) \cdot \nabla f\left(x_{0}\right)\right|
$$

uniformly for $x, x_{0} \in \Omega^{\prime}$ for any fixed choice of $k$. The rest of the proof follows as for Proposition 4.1.

Now we can now prove Corollary 1.2. Recall that, for uniformly radially tame $f$, we seek to establish the inequality

$$
\left|\int_{\Omega} e^{i \lambda f(x)}(f(x))^{\ell} \psi(x) d x\right| \lesssim \int_{\Omega} \frac{|f(x)|^{\ell} \sum_{k=0}^{m-1}\left|(x \cdot \nabla)^{k} \psi(x)\right|}{(1+|\lambda f(x)|)^{m-1}} d x
$$

with implicit constant depending only on $m, d$, the nonnegative integer $\ell$, and the constant of uniform radial tameness. Under the circumstances, it suffices to assume $\lambda=1$ since uniform radial tameness is invariant under scalar multiplication of $f$ as are the conditions (1.5)-(1.7). Following the proof, we record how to establish the inequality

$$
\int_{\Omega} \frac{|f(x)|^{\ell}}{(1+|\lambda f(x)|)^{\ell+d+1}} d x \leq C_{d}|\lambda|^{-\ell}\left|\left\{\left.x \in \Omega|| f(x)|<| \lambda\right|^{-1}\right\}\right|
$$

for the convex phase $f$ (where $d$ is the dimension: $\Omega \subset \mathbb{R}^{d}$ ), which brings (1.11) in line with the results of Bruna, Nagel, and Wainger (when we require $m \geq d+\ell+2$ ). 
Proof of Corollary 1.2. In this case, we apply the machinery of Theorem 1.1 when $\Omega$ is equal to $\mathbb{R}^{d}$. We let the indices $j$ belong to $\mathbb{Z}$ and define

$$
\Phi_{j, x}(t):=e^{3^{j} t} x
$$

for $x \neq 0$ and $\Phi_{j, 0}(0)=0$ for all $j$; in other words, the balls $B_{j}(x)$ are intervals in the ray from the origin through $x$ when $x \neq 0$ and are simply points when $x=0$. These rays and the point $\{0\}$ are exactly the leaves. The basic conditions of Theorem 1.1 are easily checked (the homogeneous space structure on the real line given by dyadic intervals centered at $x$ is well known, and the present construction is only a trivial variation). In this case, regularity of the measure is established by means of the polar coordinates formula:

$$
\int f=\int_{\mathbb{S}^{d-1}}\left[\int_{0}^{\infty} f(r \omega) r^{d-1} d r\right] d \sigma(\omega)
$$

(so we take the measure on the point $\{0\}$ to be zero). In particular, the smoothness conditions on the Jacobian hold as long as we restrict $j \leq C$ at every point for some fixed $C$ (which is an acceptable restriction from the point of view of the compatibility condition).

Now suppose that the phase $f$ is uniformly radially tame on the domain $\Omega \subset \mathbb{R}^{d}$ containing the origin. Convexity implies the inequalities

$$
f(x) \leq x \cdot \nabla f(x)
$$

for all $x \in \Omega$. Momentarily restrict attention to a single ray emanating from the origin (and if $f$ is not strictly convex on this ray, assume that we are far enough from the origin that $f \neq 0$ ). Now assuming that $f$ is uniformly radially tame to order $m \geq 2$ with constant $C$, we have

$$
\begin{aligned}
\left|(x \cdot \nabla)\left(\frac{f(x)}{x \cdot \nabla f(x)}\right)\right| & =\left|1-\frac{f(x)(x \cdot \nabla)^{2} f(x)}{(x \cdot \nabla f(x))^{2}}\right|=\left|1-\frac{f(x)\left[|x|^{2} \frac{d^{2}}{d r^{2}} f(x)+|x| \frac{d}{d r} f(x)\right]}{|x|^{2}\left(\frac{d}{d r} f(x)\right)^{2}}\right| \\
& =\left|1-\frac{f(x) \frac{d^{2}}{d r^{2}} f(x)}{\left(\frac{d}{d r} f(x)\right)^{2}}-\frac{f(x)}{x \cdot \nabla f(x)}\right| \leq C .
\end{aligned}
$$

Integrating along rays gives that

$$
\left|\frac{f(\rho x)}{\rho x \cdot \nabla f(\rho x)}-\frac{f(x)}{x \cdot \nabla f(x)}\right| \leq C \ln \rho
$$

for any $\rho>1$ (with a similar inequality when $\rho<1$ ). We define $R(x)$ (when $\nabla f(x) \neq 0)$ to be the largest integer $j$ such that

$$
3^{j} \leq \frac{1}{4 C} \frac{f(x)}{x \cdot \nabla f(x)} .
$$

By (4.4), this upper bound is at most $1 /(4 C)$, so $R(x)$ is uniformly bounded above for each $x$. Fix an $x$ and take $\rho:=e^{3^{R(x)} t}$ for $-1<t<1$. We conclude that

$$
\left|\frac{f(\rho x)}{\rho x \cdot \nabla f(\rho x)}-\frac{f(x)}{x \cdot \nabla f(x)}\right| \leq C 3^{R(x)} \leq \frac{1}{4} \frac{f(x)}{x \cdot \nabla f(x)},
$$


from which we conclude that $R(x)$ and $R(y)$ differ by at most 1 when $y$ belongs to $B_{R(x)}(x)$. The hypothesis (1.5) now follows immediately with $N=2$ by the triangle inequality (since $R(x)$ and $R(z)$ both differ by at most one from $R(z)$ when $z$ belongs to the intersection).

Next we compute:

$$
\begin{aligned}
\left|d_{x}^{k} f\right|_{R(x)} & :=\sup _{1 \leq k^{\prime} \leq k}\left|\frac{d^{k^{\prime}}}{d t^{k^{\prime}}} f\left(e^{3^{R(x)}} x\right)\right|_{t=0}\left|=\sup _{1 \leq k^{\prime} \leq k}\right| 3^{k^{\prime} R(x)}(x \cdot \nabla)^{k^{\prime}} f(x) \mid \\
& \approx \sup _{1 \leq k^{\prime} \leq k}\left|\frac{(f(x))^{k^{\prime}}}{(x \cdot \nabla f(x))^{k^{\prime}}}(x \cdot \nabla)^{k^{\prime}} f(x)\right| .
\end{aligned}
$$

By virtue of the assumption of uniform radial tameness to order $m$ (and the Leibniz rule), we can therefore conclude that $\left|d_{x}^{k} f\right|_{R(x)} \approx f(x)$ provided $k \leq m$. The condition (1.6) follows immediately. Finally, since (4.5) implies that

$$
\frac{3}{4} \frac{f(x)}{x \cdot \nabla f(x)} \leq \frac{f(y)}{x \cdot \nabla f(y)} \leq \frac{5}{4} \frac{f(x)}{x \cdot \nabla f(x)}
$$

for any $y \in B_{R(x)}(x)$, it must be the case that

$$
\rho \frac{d}{d \rho} \ln f(\rho x) \leq \frac{4}{3} \frac{x \cdot \nabla f(x)}{f(x)}
$$

for any $\rho$ with $\rho x \in B_{R(x)}(x)$, so we again integrate with respect to the variable $\rho$ to conclude that

$$
|\ln f(\rho x)-\ln f(x)| \leq \frac{4}{3} \frac{x \cdot \nabla f(x)}{f(x)}|\ln \rho| \leq \frac{1}{3 C} .
$$

Since we know $\left|d_{y} f\right|_{R(y)} \approx f(y)$, we have thus established the final hypothesis (1.7).

Having established (1.5)-(1.7), we may apply the conclusion (1.8):

$$
\left|\int_{\Omega} e^{i \lambda f(x)}(f(x))^{\ell} \psi(x) d x\right| \lesssim \int_{\Omega} \frac{\sum_{k=0}^{m-1}\left|d_{x}^{k} f^{\ell} \psi\right|_{R(x)}}{(1+\lambda f(x))^{m-1}} d x
$$

(where we have already simplified the denominator since $\left|d_{x} f\right|_{R(x)} \approx f(x)$ ). By the Leibniz rule and our estimates for $\left|d_{x}^{k} f\right|_{R(x)}$ we will have

$$
\sum_{k=0}^{m-1}\left|d_{x}^{k} f^{\ell} \psi\right|_{R(x)} \lesssim(f(x))^{\ell} \sum_{k=0}^{m-1}\left|d_{x}^{k} \psi\right|_{R(x)}
$$

(with a constant depending on $m$ and $\ell$ ). The only remaining modification is that

$$
\sum_{k=0}^{m-1}\left|d_{x}^{k} \psi\right|_{R(x)} \lesssim \sum_{k=0}^{m-1}\left|(x \cdot \nabla)^{k} \psi(x)\right|
$$

since $R(x)$ is bounded uniformly above. 
Lastly we turn our attention to (4.2). Assume $\lambda>0$ and let

$$
\begin{aligned}
& F_{0}:=\left\{x \in \Omega \mid f(x)<\lambda^{-1}\right\}, \\
& F_{k}:=\left\{x \in \Omega \mid 2^{k-1} \lambda^{-1} \leq f(x)<2^{k} \lambda^{-1}\right\}, k>0 .
\end{aligned}
$$

We have

$$
\int_{\Omega} \frac{(f(x))^{\ell}}{(1+\lambda f(x))^{d+\ell+1}} d x \leq \lambda^{-\ell} \sum_{k=0}^{\infty} \int_{F_{k}} \frac{1}{(1+\lambda f(x))^{d+1}} d x .
$$

Convexity of $f$ (and $f(0)=0$ and $\nabla f(0)=0$ ) implies that $f(\alpha x) \geq \alpha f(x)$ when $\alpha \geq 1$. In particular this means that $F_{k} \subset 2^{k} F_{0}$ for each $k$. Consequently

$$
\sum_{k=0}^{\infty} \int_{F_{k}} \frac{1}{(1+\lambda f(x))^{d+1}} d x \leq\left|F_{0}\right|+\sum_{k=1}^{\infty}\left(2^{-(k-1)}\right)^{d+1} 2^{d k}\left|F_{0}\right| .
$$

This geometric series converges and yields a finite constant for (4.2).

\subsection{Carnot-Carathéodory}

In this section we recall the framework of Street [23] and several of the results proved there. The main purpose of doing so is to establish Theorem 4.3, which exploits these results to show that Street's Frobenius theorem produces a family of balls satisfying compatibility, engulfing, weak doubling, smooth nesting, and smooth engulfing (properties (i) through (v) from the introduction). Moreover, it establishes that leaves as defined in the introduction coincide with the leaves in the Frobenius theorem. Finally, it provides an estimate for the smoothness of the Jacobian when integrating Lebesgue measure on a leaf. This does not explicitly prove the regularity of measure hypothesis, since we will need a global measure $\mu$ which factors as Lebesgue measure (up to smooth density) on the leaves. It appears that such a measure may not exist in certain exceptionally pathological cases. However, if the dimension of the leaves is constant on some neighborhood, then the classical coarea formula combines with the Jacobian estimate in Theorem 4.3 to guarantee that the $d$-dimensional Lebesgue measure factors locally in exactly the way required by regularity of measure. Beyond this, as was already seen in the previous section (near the origin), it is often possible to establish regularity of measure directly even when the dimension of the leaves is not constant.

We now recall the framework of Street [23]. Begin with a finite collection $X_{1}, \ldots, X_{q}$ of $C^{1}$ vector fields on $\Omega$. Each vector field has a nonzero "formal degree" $d_{1}, \ldots, d_{q}$ belonging to $[0, \infty)^{d}$. Fix some compact subset $K \subset \Omega$ and $\xi \in(0,1]^{d}$. Suppose that for any $x_{0} \in K$ and any $a=\left(a_{1}, \ldots, a_{q}\right) \in\left(L^{\infty}([0,1])\right)^{q}$ with $\|\left.\sqrt{\sum_{i=1}^{q}\left|a_{i}\right|^{2}}\right|_{L^{\infty}}<1$, the ordinary differential equation

$$
\gamma^{\prime}(t)=\sum_{i=1}^{q} \xi^{d_{i}} a_{i}(t) X_{i}(\gamma(t))
$$

has a (weak) solution on $[0,1]$ with initial condition $\gamma(0)=x_{0}$. 
Next fix a set $A \subset\left\{\delta \in[0,1]^{d} \mid \delta \neq 0, \delta \leq \xi\right\}$. This set $A$ represents the allowable multiscales $\delta$ (where "allowable" is in principle determined by the context in which the Carnot-Carathéodory machinery is being applied). For present purposes, we add a constraint to the collection $A$ of allowable $\delta$; we assume that $\delta=\left(\delta_{1}, \ldots, \delta_{d}\right) \in A$ implies $\left(\epsilon \delta_{1}, \ldots, \epsilon \delta_{d}\right) \in A$ for any $\epsilon \in(0,1)$. Note that this isotropic dilation condition on $A$ will generally be true in applications of interest; in particular it holds in the case of "weak comparability." The CarnotCarathéodory ball $B_{(X, d)}\left(x_{0}, \delta\right)$ is defined as the set $y \in \Omega$ such that there exists an $a \in\left(L^{\infty}([0,1])\right)^{q}$ with $\|\left.\sqrt{\sum_{i=1}^{q}\left|a_{i}\right|^{2}}\right|_{L^{\infty}}<1$ as before such that the necessarily unique solution of

$$
\gamma^{\prime}(t)=\sum_{i=1}^{q} \delta^{d_{i}} a_{i}(t) X_{i}(\gamma(t))
$$

with $\gamma(0)=x_{0}$ has $\gamma(1)=y$.

The assumptions begin with the integrability condition: for every $\delta \in A$ and $x \in K$, it must be the case that

$$
\left[\delta^{d_{i}} X_{i}, \delta^{d_{i^{\prime}} X_{i^{\prime}}}\right]=\sum_{k=1}^{q} c_{i, i^{\prime}}^{k, \delta, x} \delta^{d_{k}} X_{k}
$$

at every point $y \in B_{(X, d)}(x, \delta)$. Next assume that for some $m \geq 2$, the vector fields are $C^{m}$ on $B_{(X, d)}(x, \xi)$ for every $x \in K$, that $X^{\alpha} c_{i, i^{\prime}}^{k, \delta}$ is continuous on this same ball whenever $|\alpha| \leq m$, and

$$
\begin{array}{r}
\sup _{x \in K}\left\|X_{i}\right\|_{C^{m}\left(B_{(X, d)}(x, \xi)\right)} \\
\sup _{\substack{\delta \in A \\
x \in K}} \sum_{|\alpha| \leq m}\left\|(\delta X)^{\alpha} c_{i, i^{\prime}}^{k, \delta, x}\right\|_{C^{0}\left(B_{(X, d)}(x, \xi)\right)}<\infty .
\end{array}
$$

(Note that the norms are taken with respect to some implicit, fixed coordinate system on $\Omega$.) For each $x \in K$, let $n_{0}(x)$ be the dimension of the span of $X_{1}, \ldots, X_{q}$ at $x$. For each $\delta \in A$, Street (in agreement with Nagel, Stein, and Wainger) identifies an appropriate subcollection $J(x, \delta) \subset\{1, \ldots, q\}$ and defines a mapping on a neighborhood of the origin in $\mathbb{R}^{J(x, \delta)}$ by

$$
\Phi_{x, \delta}(u):=\exp \left(\sum_{i \in J(x, \delta)} u_{i} \delta^{d_{i}} X_{i}\right) x .
$$

Theorem 6.4 (Street [23]). There are $m$-admissible constants $\rho$ and $r_{2}<r_{1}$ such that the following hold for all $\delta \in A$ and $x \in K$ :

- $B_{(X, d)}(x, \rho \delta) \subset \Phi_{x, \delta}\left(\mathbb{B}^{n_{0}(x)}\left(r_{2}\right)\right) \subset \Phi_{x, \delta}\left(\mathbb{B}^{n_{0}(x)}\left(r_{1}\right)\right) \subset B_{(X, d)}(x, \delta)$.

- $\Phi_{x, \delta}(u)$ is one-to-one on $\mathbb{B}^{n_{0}(x)}\left(r_{1}\right)$

- If $Y_{i}$ is the pullback of $\delta^{d_{i}} X_{i}$ under the map $\Phi_{x, \delta}$ on the ball $\mathbb{B}^{n_{0}(x)}\left(r_{1}\right)$, then $\left\|Y_{i}\right\|_{C^{m}} \lesssim 1$. Furthermore,

$$
\left(Y_{J(x, \delta)_{1}}, \ldots, Y_{J(x, \delta)_{n_{0}(x)}}\right)=(I+B(x, u)) \nabla_{u}
$$

for some $C^{m}$ matrix $B(x, u)$ of norm at most $1 / 2$. 
- For all $u \in B^{n_{0}(x)}\left(r_{1}\right),\left|\operatorname{det}_{n_{0}(x) \times n_{0}(x)} d \Phi_{x, \delta}(u)\right| \approx\left|\operatorname{det}_{n_{0}(x) \times n_{0}(x)} \delta X(x)\right|$.

Here $\delta X$ is the matrix with columns $\delta^{d_{i}} X_{i}$ and $\operatorname{det}_{k \times k}$ is the vector whose entries are the determinants of all $k \times k$ minors of that matrix. An m-admissible constant is one which depends only on upper bounds of (4.6), (4.7), the dimension of $\Omega, q, d$, lower bounds for the coordinates of $\xi$, and upper and lower bounds for the coordinates of $\sum d:=\left(\sum_{i=1}^{d} d_{1}^{i}, \ldots, \sum_{i=1}^{d} d_{q}^{i}\right)$.

We now come to the final theorem, which is essentially a repackaging of a number of Street's definitions and estimates to illustrate that he implicitly constructed a space of exactly the sort we have defined in the present work.

Theorem 4.3. Under the same hypotheses as those (described above) for Theorem 6.4 of [23], define balls $B_{j}(x):=\Phi_{x, M^{j}}\left(\mathbb{B}^{n_{0}(x)}\left(r_{1}\right)\right)$ and homeomorphisms $\Phi_{j, x}(u):=\Phi_{x, M^{j}}\left(r_{1}^{-1} u\right)$ when $M^{j} \in A$. There is a choice of constant $M$ such that the hypotheses (i) through (v) of Theorem 1.1 are satisfied when the indices $j$ are restricted to have $M^{j} \in A$ and each component of $j$ sufficiently negative. Furthermore, the leaves of the foliation given by the Frobenius theorem are leaves in the sense of Theorem 1.1, and

$$
\frac{1}{\operatorname{Vol}\left(B_{j}(x)\right)} \int_{B_{j}(x)} f d \mu_{L}=\int_{\mathbb{B}} f \circ \Phi_{j, x}(t) J_{j, x}(t) d t
$$

when $\mu_{L}$ is the induced Lebesgue measure on the leaf $L$ for some nonnegative function $J_{j, x}$ with $J_{j, x} \approx 1$ and $\left\|J_{j, x}\right\|_{C^{m}} \lesssim 1$.

Proof. Let $M$ be some constant greater than one to be determined momentarily. For suitable $j \in \mathbb{Z}^{d}$ we define $B_{j}(x):=\Phi_{x, M^{j}}\left(\mathbb{B}^{n_{0}(x)}\left(r_{1}\right)\right)$ and $\Phi_{j, x}(u):=$ $\Phi_{x, M^{j}}\left(r_{1}^{-1} u\right)$. We will first show that this system satisfies the axioms (i) through (vi). In this case, the compatibility condition (i) is trivially satisfied because the set $A$ of admissible $\delta$ has not been taken to depend on $x$ and it has explicitly been assumed to be closed under contractions $\delta \mapsto M^{-1} \delta$. It is essentially a matter of understanding definitions to show that when $\delta^{\prime} \leq \delta$

$$
B_{(X, d)}\left(y, \delta^{\prime}\right) \cap B_{(X, d)}(x, \delta) \neq \emptyset \Longrightarrow B_{(X, d)}\left(y, \delta^{\prime}\right) \subset B_{(X, d)}\left(x, 2^{p} \delta\right)
$$

for any $p$ with $p \sum_{i=1}^{d} d_{k}^{i} \geq 1$ for all $k=1, \ldots, q$. This follows easily from concatenating paths and rescaling; note that $p$ is an admissible constant in Street's terminology. As long as $M^{-1} \leq 2^{-p} \rho$ we have

$$
\begin{aligned}
B_{j}(x) \cap B_{j^{\prime}}(y) \neq \emptyset & \Longrightarrow \quad B_{(X, d)}\left(x, M^{j}\right) \cap B_{(X, d)}\left(y, M^{j^{\prime}}\right) \neq \emptyset \\
& \Longrightarrow \quad B_{j^{\prime}}(y) \subset B_{(X, d)}\left(y, M^{j^{\prime}}\right) \subset B_{(X, d)}\left(x, 2^{p} M^{j}\right) \\
& \subset B_{(X, d)}\left(x, \rho M^{j+1}\right) \subset B_{j+1}(x) .
\end{aligned}
$$

To establish weak doubling, we use the doubling condition from Corollary 6.4 of [23]; namely,

$$
\operatorname{Vol}\left(B_{(X, d)}(x, 2 \delta)\right) \lesssim \operatorname{Vol}\left(B_{(X, d)}(x, \delta)\right)
$$

for $\delta$ sufficiently small, where Vol represents the $n_{0}(x)$-dimensional Hausdorff measure. 
We know explicitly from Street's paper that the measure of such a ball is never zero nor infinity when the entries of $\delta$ are nonzero. It is straightforward to see that the nesting property guarantees that the doubling property holds for the dyadic balls $B_{j}(x)$ as well. Now suppose $B_{j}\left(x_{1}\right), \ldots, B_{j}\left(x_{N}\right)$ are mutually disjoint and that some ball $B_{j+1}(x)$ intersects all of the balls $B_{j+1}\left(x_{k}\right)$. Then we have the containments $B_{j}(x) \subset B_{j+2}\left(x_{k}\right)$ and $B_{j}\left(x_{k}\right) \subset B_{j+2}(x)$ for each $k$. But now the observations

$$
\begin{aligned}
\sum_{k=1}^{N} \operatorname{Vol}\left(B_{j}\left(x_{k}\right)\right) & \leq \operatorname{Vol}\left(B_{j+2}(x)\right), \\
\operatorname{Vol}\left(B_{j+2}(x)\right) & \approx \operatorname{Vol}\left(B_{j}(x)\right) \leq \operatorname{Vol}\left(B_{j+2}\left(x_{k}\right)\right) \approx \operatorname{Vol}\left(B_{j}\left(x_{k}\right)\right)
\end{aligned}
$$

combine to give the uniform inequality

$$
N \operatorname{Vol}\left(B_{j+2}(x)\right) \lesssim \operatorname{Vol}\left(B_{j+2}(x)\right)
$$

which, in turn, gives a uniform upper bound on $N$ because the volume is known to be nonzero.

Now we analyze the smooth structures. The smooth nesting property follows immediately from Theorem 6.4 of [23] with $c:=r_{2} / r_{1}$. Smooth engulfing is almost equally immediate. Given two balls $B_{j}(x)$ and $B_{j^{\prime}}\left(x^{\prime}\right)$ of comparable scale with a nontrivial intersection, there will be a third ball $B_{j^{\prime \prime}}\left(x^{\prime \prime}\right)$ of another comparable scale that contains them both. Now

$$
\Phi_{x, \delta}^{-1} \circ \Phi_{x^{\prime}, \delta^{\prime}}=\left(\Phi_{x^{\prime \prime}, \delta^{\prime \prime}}^{-1} \circ \Phi_{x, \delta}\right)^{-1} \circ\left(\Phi_{x^{\prime \prime}, \delta^{\prime \prime}}^{-1} \circ \Phi_{x^{\prime}, \delta^{\prime}}\right) .
$$

On this third ball, we have by pullbacks that

$$
\Phi_{x, \delta}^{-1} \circ \Phi_{x^{\prime}, \delta^{\prime}}(u)=\exp \left(\sum_{i \in J\left(x^{\prime}, \delta^{\prime}\right)} u_{i}\left(\delta^{\prime}\right)^{d_{i}}\left(\delta^{\prime \prime}\right)^{-d_{i}} Y_{i}^{\prime \prime}\right) \Phi_{x, \delta}^{-1}\left(x^{\prime}\right) .
$$

We know that the pullback vector fields $Y_{i}^{\prime \prime}$ of $\left(\delta^{\prime \prime}\right)^{d_{i}} X_{i}$ via $\Phi_{x^{\prime \prime}, \delta^{\prime \prime}}$ are uniformly in $C^{m}$, so the mapping $\Phi_{x, \delta}^{-1} \circ \Phi_{x^{\prime}, \delta^{\prime}}(u)$ must be uniformly $C^{m}$ as well. Moreover, if we choose $x^{\prime \prime}=x$, we will have that $\left(\Phi_{x^{\prime \prime}, \delta^{\prime \prime}}^{-1} \circ \Phi_{x, \delta}\right)^{-1}$ will also be uniformly in $C^{m}$ because of the comparability of Jacobians:

$$
\begin{aligned}
\left|\underset{n_{0}(x) \times n_{0}(x)}{\operatorname{det}} d \Phi_{x, \delta}(u)\right| & \approx\left|\underset{n_{0}(x) \times n_{0}(x)}{\operatorname{det}_{1}} \delta X(x)\right| \approx\left|\underset{n_{0}(x) \times n_{0}(x)}{\operatorname{det}} \delta^{\prime \prime} X(x)\right| \\
& \approx\left|\operatorname{det}_{n_{0}(x) \times n_{0}(x)} d \Phi_{x, \delta^{\prime \prime}}\left(u^{\prime \prime}\right)\right| ;
\end{aligned}
$$

this implies that the Jacobian determinant of $\Phi_{x^{\prime \prime}, \delta^{\prime \prime}}^{-1} \circ \Phi_{x, \delta}$ is bounded uniformly above and below, and Cramer's rule then implies that the inverse mapping will be uniformly in $C^{m}$ depending on the $C^{m}$ norm of the mapping $\Phi_{x^{\prime \prime}, \delta^{\prime \prime}}^{-1} \circ \Phi_{x, \delta}$ itself.

Finally, regarding the foliations, leaves, and measures, see appendices B and $\mathrm{C}$ of [23]. In particular, we have the formula

$$
\int_{B_{j}(x)} f d \mu_{L}=\int f \circ \Phi_{j, x}(t)\left|\operatorname{det}_{n_{0}(x) \times n_{0}(x)}(d \Phi(t))\right| d t,
$$


where $|\cdot|$ is the usual Euclidean length. The magnitude of $\left|\operatorname{det}_{n_{0}(x) \times n_{0}(x)}(d \Phi(t))\right|$ is shown by Street to be uniformly bounded above and below by the volume of the ball. To show smoothness, we use Lemma 4.16 and Proposition 4.17 of [23], which together show that

$$
\frac{\left|\operatorname{det}_{n_{0}(x) \times n_{0}(x)} d \Phi_{x, \delta}(u)\right|}{\left|\operatorname{det}_{n_{0}(x) \times n_{0}(x)} \delta X\left(\Phi_{x, \delta}(u)\right)\right|}
$$

is uniformly in $C^{m}$ on the ball. Since the pullback vector fields $Y_{i}$ are uniformly in $C^{m}$ and uniformly span (meaning that there is an $n_{0}(x)$-tuple which when grouped into a matrix is uniformly close to the identity matrix, and in particular, has determinant uniformly bounded below), it suffices to show that

$$
\left|(\delta X)_{i_{1}} \cdots(\delta X)_{i_{k}} \operatorname{det}_{n_{0}(x) \times n_{0}(x)}(\delta X)(x)\right| \lesssim\left|\operatorname{det}_{n_{0}(x) \times n_{0}(x)}(\delta X)(x)\right|
$$

uniformly for any choice of $i_{1}, \ldots, i_{k}$ with $k \leq m$. From the proof of Lemma 4.6 in [23], we see that we can write

$$
(\delta X)_{i_{k}} \operatorname{det}_{n_{0}(x) \times n_{0}(x)}(\delta X)(x)
$$

as some smooth matrix with admissible norm times $\operatorname{det}_{n_{0}(x) \times n_{0}(x)}(\delta X)(x)$ itself (admissible because the smooth functions appearing are literally those bounded by (4.6) and (4.7)). The final result follows by induction on $k$.

\section{References}

[1] Bruna, J., Nagel, A. And Wainger, S.: Convex hypersurfaces and Fourier transforms. Ann. of Math. (2) 127 (1988), no. 2, 333-365.

[2] Carbery, A.: A uniform sublevel set estimate. In Harmonic analysis and partial differential equations, 97-103. Contemp. Math. 505, Amer. Math. Soc., Providence, RI, 2010.

[3] Carbery, A., Christ, M. and Wright, J.: Multidimensional van der Corput and sublevel set estimates. J. Amer. Math. Soc. 12 (1999), no. 4, 981-1015.

[4] Carbery, A. And Wright, J.: What is van der Corput's lemma in higher dimensions? Proceedings 6th International Conference on Harmonic Analysis and Partial Differential Equations (El Escorial, 2000). Publ. Mat., vol. extra (2002), 13-26.

[5] Coifman, R. R. And Weiss, G.: Analyse harmonique non-commutative sur certains espaces homogènes. Étude de certaines intégrales singulières. Lecture Notes in Mathematics 242, Springer-Verlag, Berlin, 1971.

[6] Collins, T., Greenleaf, A. and Pramanik, M.: A multi-dimensional resolution of singularities with applications to analysis. Amer. J. Math. 135 (2013), no. 5, 1179-1252.

[7] Cowling, M. and Mauceri, G.: Oscillatory integrals and Fourier transforms of surface carried measures. Trans. Amer. Math. Soc. 304 (1987), no. 1, 53-68.

[8] Greenblatt, M.: An elementary coordinate-dependent local resolution of singularities and applications. J. Funct. Anal. 255 (2008), no. 8, 1957-1994. 
[9] Greenblatt, M.: Resolution of singularities in two dimensions and the stability of integrals. Adv. Math. 226 (2011), no. 2, 1772-1802.

[10] Gressman, P. T.: Uniform estimates for cubic oscillatory integrals. Indiana Univ. Math. J. 57 (2008), no. 7, 3419-3442.

[11] Gressman, P. T.: Uniform geometric estimates of sublevel sets. J. Anal. Math. 115 (2011), 251-272.

[12] Ikromov, I., Kempe, M. And Müller, D.: Estimates for maximal functions associated with hypersurfaces in $\mathbb{R}^{3}$ and related problems of harmonic analysis. Acta Math. 204 (2010), no. 2, 151-271.

[13] Iosevich, A.: Fourier transform, $L^{2}$ restriction theorem, and scaling. Boll. Unione Mat. Ital. Sez. B Artic. Ric. Mat. (8) 2 (1999), no. 2, 383-387.

[14] Karpushinin, V. N.: A theorem concerning uniform estimates of oscillatory integrals when the phase is a function of two variables. J. Soviet. Math. 35 (1986), 2809-2826.

[15] Nagel, A., Stein, E. M. And Wainger, S.: Balls and metrics defined by vector fields. I. Basic properties. Acta Math. 155 (1985), no. 1-2, 103-147.

[16] Phong, D. H. And Stein, E. M.: Damped oscillatory integral operators with analytic phases. Adv. Math. 134 (1998), no. 1, 146-177.

[17] Phong, D. H., Stein, E. M. And Sturm, J. A.: On the growth and stability of real-analytic functions. Amer. J. Math. 121 (1999), no. 3, 519-554.

[18] Phong, D. H., Stein, E. M. And Sturm, J. A.: Multilinear level set operators, oscillatory integral operators, and Newton polyhedra. Math. Ann. 319 (2001), no. 3, 573-596.

[19] Phong, D. H. And Sturm, J.: Algebraic estimates, stability of local zeta functions, and uniform estimates for distribution functions. Ann. of Math. (2) 152 (2000), no. 1, 277-329.

[20] Pramanik, M. And Yang, C. W.: Decay estimates for weighted oscillatory integrals in $\mathbb{R}^{2}$. Indiana Univ. Math. J. 53 (2004), no. 2, 613-645.

[21] Rogers, K. M.: Sharp van der Corput estimates and minimal divided differences. Proc. Amer. Math. Soc. 133 (2005), no. 12, 3543-3550 (electronic).

[22] Seeger, A.: Radon transforms and finite type conditions. J. Amer. Math. Soc. 11 (1998), no. 4, 869-897.

[23] Street, B.: Multi-parameter Carnot-Carathéodory balls and the theorem of Frobenius. Rev. Mat. Iberoam. 27 (2011), no. 2, 645-732.

[24] TaO, T. And Wright, J.: $L^{p}$ improving bounds for averages along curves. J. Amer. Math. Soc. 16 (2003), no. 3, 605-638.

[25] VArČenko, A. N.: Newton polyhedra and estimates of oscillatory integrals. Funkcional. Anal. i Priložen. 10 (1976), no. 3, 13-38.

Received March 4, 2013.

Philip T. Gressman: David Rittenhouse Laboratory, 209 South 33rd Street, Philadelphia, PA 19104, USA.

E-mail: gressman@math.upenn.edu

Partially supported by NSF grant DMS-1101393 and an Alfred P. Sloan Foundation Fellowship. 